\title{
How Cultural Norms Influence Persuasive Design: A Study on Chinese Food Journaling Apps
}

\author{
$\mathrm{Xi} \mathrm{Lu}$ \\ Informatics, University of California \\ Irvine \\ xlu30@uci.edu
}

\author{
Yunan Chen \\ Informatics, University of California \\ Irvine \\ yunanc@ics.uci.edu
}

\author{
Daniel A. Epstein \\ Informatics, University of California \\ Irvine \\ epstein@ics.uci.edu
}

\begin{abstract}
Persuasive features are often used in food journaling apps to help people reach various personal goals. Our understanding of persuasive design in food journaling apps has primarily been built from studying and designing apps in Western contexts. However, varied cultural perceptions around journaling goals, such as healthy eating and weight management, suggest that the design of persuasive features may differ across cultures. We therefore investigate how culture influences the use of persuasive techniques in Chinese food journaling apps and consequently people's journaling experiences. Through reviewing features of Chinese apps and interviewing people who have used them, we find that some Chinese apps heavily emphasize body shape, and people's motivations for journaling and desires for journaling apps similarly focus more on body shape than diet. We discuss tensions and opportunities for food journaling apps which align with or account for cultural norms while challenging unhealthy or problematic aspects of them.
\end{abstract}

\section{CCS CONCEPTS}

- Human-centered computing; • Human computer interaction $(\mathrm{HCI}) ; \cdot$ Empirical studies in $\mathrm{HCI}$;

\section{KEYWORDS}

food journaling, cultural influences, personal informatics, selftracking

\section{ACM Reference Format:}

Xi Lu, Yunan Chen, and Daniel A. Epstein. 2021. How Cultural Norms Influence Persuasive Design: A Study on Chinese Food Journaling Apps. In Designing Interactive Systems Conference 2021 (DIS '21), June 28-7uly 02, 2021, Virtual Event, USA. ACM, New York, NY, USA, 19 pages. https: //doi.org/10.1145/3461778.3462142

\section{INTRODUCTION}

Food journaling has been an area of focus in HCI research, looking to understand the diversity of user's needs when tracking food [7, 49, 50], explore the current challenges of journaling food $[16,18,22]$, and develop innovative tools to remove barriers for tracking food [17, 25, 34]. Through continuously tracking of dietary information, food journaling apps can help people understand their

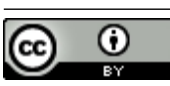

This work is licensed under a Creative Commons Attribution International 4.0 License.

DIS '21, fune 28- July 02, 2021, Virtual Event, USA

(c) 2021 Copyright held by the owner/author(s).

ACM ISBN 978-1-4503-8476-6/21/06.

https://doi.org/10.1145/3461778.3462142 eating patterns, manage their weight, or build a healthier diet [18]. However, many food journaling goals require long-term effort and engagement, and supporting people in sticking to their intended behaviors is therefore a common goal for food journaling apps.

Persuasive technology is technology that leverages design strategies to change people's attitudes or behaviors [30,58]. The persuasive lens has been widely applied in HCI to design technology to support people in making positive behavioral choices, often towards promoting health and wellness [32, 60, 63]. Common persuasive design features, such as goal setting, reminders, rewards, and social support, have been shown to facilitate behavior change by increasing and sustaining engagement. For example, food journals sending daily messages giving feedback on weight loss process have been shown to increase engagement and efficiency in weight loss programs over traditional paper diaries [10]. To support making better food decisions, another food journaling app leveraged persuasive techniques including self-monitoring and rewards to help people overcome their cravings for snacks [38]. Despite these benefits, prior studies have shown that inappropriate persuasive designs could also lead to unintended negative consequences. For example, numeric goals can make food journalers to become obsessed with counting calories [32]. Persuasive feedback provided by apps could make people feel guilty about not completing their goals, and make people feel that the app is trying to control their eating habits [37]. Eikey et al. [21] found that food journaling apps are often used by people with Eating disorders (ED), but could exacerbate ED symptoms by enabling people to set unhealthy weight loss targets or daily calorie budgets.

Although we have built up some understanding of how persuasive techniques can be incorporated into food journaling apps and how people perceive them, most of our understanding has come from studying and designing with people in Western contexts. While people use food journaling apps worldwide typically for either diet- or weight-related goals [18], cultures vary in how they perceive what constitutes a healthy diet and weight. In this study, we therefore explore how cultural norms influence the use of persuasive design in food journaling apps designed outside of Western contexts, specifically food journaling apps in China. Compared with many Western countries, the eating culture in China is more vegetarian-oriented [39].In Chinese culture, people often expect that the foods they eat will not only satisfy hunger, but also improve health, cure diseases, and help them socially connect with others [45]. To strengthen interpersonal relationships, Chinese food culture prefers to share dishes, while Western culture tends to serve food individually [52]. Compared with Western culture, China has more acceptance of a thin ideal, which regards thinness as the perfect body shape, as a social norm [42, 76]. These cultural 
differences suggest potentially different design needs for food journaling technology and offer a case study into how cultural norms influence persuasive design.

Building on a preliminary analysis of common features in Chinese and Western food journaling apps and pilot interviews, we particularly found that features in Chinese apps frequently emphasized body shape, and people were often more interested in journaling their food to achieve a thin body shape rather than follow a particular diet. We therefore conducted semi-structured interviews with 18 Chinese participants to understand their perceptions of persuasive design elements in Chinese food journaling apps that emphasized body image and their counterparts in Western apps. We also analyzed the features of 9 popular Chinese and 11 Western food journal apps regarding its persuasive features centered on body images according to Oinas-Kukkonen and Harjumaa's [58] framework for persuasive systems design. We found that cultural norms around weight and beauty led participants to pursue body image over a healthy diet, and Chinese apps leveraged persuasive strategies which aligned with this norm. Participants who held this cultural beauty norm were effectively supported by persuasive strategies that portrayed ideal body shape, while participants who held different norms felt excluded by the technology.

This work contributes:

A systematic examination on how culture influences the persuasive design of Chinese food journaling apps by comparing persuasive strategies leveraged by Chinese apps and their Western counterparts. Shaped by the cultural beauty norm of pursuing thinness, Chinese apps tended to utilize persuasive design principles to portray a thin ideal. Meanwhile, Western apps leveraged some of these persuasive techniques to help people lose weight and become healthier.

An empirical understanding of how Chinese people perceive the persuasive strategies used by Chinese food journaling apps to emphasize body image. Participants whose food journaling goals aligned with the cultural beauty norm felt positive about the use of persuasive strategies because they were motivational, instructive, and provide more information than just food in the app. Such design, however, could trigger anxiety, lack credibility, and reinforce misleading knowledge about body shape and weight loss among some users.

Design opportunities for critically utilizing persuasive strategies in food journaling apps to avoid consequences resulting from designs aligning with a single cultural ideology. To help people pursue a healthier and more inclusive food goals, we suggest that technology can leverage persuasive techniques to help people appreciate different types of healthy body shapes and to educate people about healthy eating practices and healthy bodies. In addition, persuasive principles can be used to help build a more positive and celebratory relationship between healthy food and healthy body shape.

\section{RELATED WORK}

We examine how culture influences the use of persuasive design in Chinese food journaling apps and how people in China perceived these design elements. We first provide background knowledge on food journaling practices and technology. We also touch upon persuasive design and its application in HCI, since our study applies the framework for persuasive systems design to examine Chinese and Western food journaling apps. We also introduce previous HCI literature exploring cultural influence on designing technology. To contextualize our findings about the use of persuasive design strategies to emphasize body image in Chinese food journaling apps, we briefly describe cultural norms around beauty and body image.

\subsection{Food Journaling Practices and Technology}

Food journaling is a popular health tracking domain in research, being among the most popular domains of study within personal informatics literature [24], and commercially, with $42 \%$ of U.S. adults having tried an app for diet or nutrition tracking as of 2017 [1]. People track food for various reasons including losing weight, developing a healthier diet, and identifying foods they are allergic to $[18,40]$.

Studies of food journaling interventions have revealed that food tracking can influence people both positively and negatively. Food journaling apps have been shown to effectively support people's goals of managing weight or attaining a healthier diet $[16,18]$. Beyond these benefits, previous studies indicate that food journaling may also benefit people in addressing physical or mental health issues. For example, Barbarin et al. [7] found that food trackers can help female users cope with emotion- and stress-related eating issues through setting healthy goals, sustaining motivation, and emotional support. Ayobi et al. [6] found that both paper journals and food tracking apps can improve health and wellness of people with multiple sclerosis through mindful eating. However, food tracking tools sometimes exacerbate people's unhealthy eating behaviors. Cordeiro et al. [18] examined common challenges in food tracking practice, finding that barriers to food entry may nudge users to eat foods that are contrary to their goal, such as logging packaged foods over healthy homemade foods to reduce logging burden. Furthermore, people felt they were judged by their food journaling apps when eating too much or consuming unhealthy food, making them feel guilty or even causing them to stop journaling. Studies of people with eating disorders have shown that food journaling apps may exacerbate unhealthy eating behaviors by causing obsession with counting calories, setting unhealthy goalbased plans, or providing negative feedback when a person eats more than planned $[22,23,44]$.

\subsection{Persuasive Design and Cultural Influence in $\mathrm{HCI}$}

Persuasive technologies are information technologies that aim to persuade users to change their attitudes or behaviors and enact some change [30,58]. Oinas-Kukkonen and Harjumaa [58] further categorized three types of influence that persuasive technologies can have on people's attitude or behavior: reinforcement, change, and shaping. Technology that provides reinforcement strengthens people's current attitudes or behaviors, technology supporting change alters people's current attitudes or behaviors, and technology encouraging shaping helps formulate a new attitude or behavior.

Persuasive design strategies have often been leveraged in food journaling technology. For example, Aydin et al. [5] developed an app that encourages people to consume food before its expiration 
date by positively and negatively reinforcing the behavior, rewarding users for good food usage behavior, notifying and presenting food that will soon expire, and punishing people by removing points when food is wasted. Hsu et al. [38] designed a persuasive app to help people overcome their cravings for snacks, supporting a person in tracking moments where they are craving snacks alongside moments where they forewent having a snack to give them a sense of self-achievement. Moreover, when tracking cravings, the app prompted users with a task designed to reduce cravings to further aim to persuade behavior.

Persuasive design also sometimes aims to nudge people to perform behaviors without their knowledge or support [63]. Inappropriate and ill-thought-out use of persuasive designs may produce unexpected negative influences on people. Thomson et al. [70] argue that the fragmented application of persuasive principles without considering what the comprehensive effect that combined persuasive designs will together produce does not support diverse people's diverse needs, and leads to inappropriate use of notifications, reminders, and feedback. Young people reported the persuasive nature of healthy eating and fitness apps made them feel guilty when they did not complete their goals, and thus made them feel as though they were controlled by the app [37]. The persuasive feature of providing numeric goals and rewards can make people become obsessed with this feedback, leading people to prefer particular activities over some others because they can be tracked and engaging in activities explicitly to gain rewards [32]. Considering the nature of persuasive design, Purpura et al. [63] argued that the values and ethical issues of persuasive computing need to be carefully considered because persuasion could be coercive when users are provided with designers' viewpoints around ideal health and wellbeing without the chance to choose their own.

Previous HCI studies have typically examined how culture influences the design of interfaces from the visual perspective, such as color, graphic elements, textual components, and information architecture [53, 62, 65] or usability, such as people's informationseeking behaviors [26]. For example, Chinese participants preferred interfaces with more graphic elements, while Western participants tended to engage with interfaces with more textual information $[15,35]$. Faiola et al. [26] find that people's ability to seek information in interfaces may be influenced by their culturally-shaped cognitive style of processing information, finding it easier to locate information in interfaces designed by people with the same cultural background. Wanick et al. [72] explored persuasion in advergaming design, suggesting that the design of persuasive messages should be tailored to the target user's cultural background. In our work, we explore beyond the visual aspects that are often explored in previous HCI studies [53, 62, 65] to understand how the features of applications, specifically persuasive features in food journaling applications, may be influenced by culture.

\subsection{Body Image and Cultural Beauty Norms}

Body image refers to how an individual perceives his or her own body [28, 67]. It is dynamically constructed in people's daily experiences and situated socio-cultural norms [67]. Previous studies have pointed out that food journaling apps can be appropriated to reinforce body image concerns, such as people with ED using apps to limit their weight $[21,23]$.
Concerns around body image are typically caused by cultural beauty principles, such as the concept of a thin ideal body shape, which suggests that the ideal woman possesses a slender physique [36]. While the thin ideal is prevalent in both Western and Eastern cultures, studies show that people in Eastern culture tend to be more accepting of the thin ideal as a social norm [42]. People in Eastern culture overall have a lower body satisfaction and practice dieting behaviors more frequently than people in Western countries $[20,41,54]$. In China, the cultural beauty norm is to pursue thinness, especially for females [76]. For example, there is a famous Chinese saying, “Beautiful girls shall never weigh over $50 \mathrm{~kg} / 110 \mathrm{lbs}$ (好女不过 百)”. Popular trends that promote this cultural beauty norm can be often seen in Chinese social media, such as the A4-waist challenge (A4腰挑战) where females held a piece of A4-sized paper in front of them to show how thin their waists are [75]. While the thin ideal is deeply rooted in East Asian culture, such as China, little is known about how this cultural norm has influenced the design of food journaling apps and the way people perceive food journaling apps in this particular socio-cultural context.

\section{DATA COLLECTION AND ANALYSIS}

We conducted three research activities to explore the cultural influence on the use of persuasive strategies in Chinese apps as well as understanding Chinese participants' attitudes toward these persuasive techniques. We first performed a preliminary app review to compare Chinese and Western food journaling apps and also conducted pilot interviews. From this, we found some predominant features in Chinese apps were associated with body image and people's motivation for using food journaling apps also focused more on body shape rather than diet. We therefore selected some app screenshots that Chinese apps tend to differ from Western apps, including features that emphasize body image, to be used in our formal interview. We then conducted semi-structured interviews with 18 participants (Table 2). The interview was mainly to understand participants' perceptions toward features that emphasize body shape as well as their in-situ food journaling experiences related to body image. At last, as we realized the persuasive nature of features used in Chinese apps to portray the perfect body shape, we leveraged the framework for persuasive systems design [58] to label any design features in Chinese food journaling apps that were about body image. As a comparison, we also checked how these persuasive strategies were implemented in Western apps.

Reflecting on our positionality as researchers, two of the authors are from China, the third from the United States. All authors were living and working in the United States at the time of writing. Although we did not intend to judge the benefits and limitations of either Western food journaling apps or Chinese ones, our cultural background influences our perspectives and opinions on how apps in each setting are designed and how we have reported on those choices. Past work has highlighted disadvantages of both typical approaches (e.g., calorie-counting, body shape). Focus on calorie intake in mobile apps has been shown to lead some people to become addicted to monitoring their calories [21, 37] and can promote unhealthy eating behaviors, especially for people with ED $[21,44]$. Technology focusing on focus on body image and a thin 
Table 1: We reviewed the features of 9 Chinese apps and 11 Western apps for food journaling, filtering by popularity

\begin{tabular}{lll}
\hline Region & $\begin{array}{l}\text { Selection } \\
\text { Criteria }\end{array}$ & Apps selected (ranked from most to least reviewed) \\
\hline Chinese & $>1,000$ & Mint; So Light; Change; iCalorie; Weight loss Secretary; Light+; Copenhagen Method to Lose Weight; Food \\
apps & reviews & Storehouse; Good body knowledge \\
Western & $>7,500$ & WW(Weight Watchers); MyFitnessPal; Lose It!-Calorie Counter; Carb Manager: Keto Diet App; Noom; \\
apps & reviews & Lifesum: Diet \& Macro Tracker; Fooducate Nutrition Tracker; Keto.app-Keto Diet Tracker; iTrackBites: \\
& & Track Weight Loss; MyPlate Calorie Counter; MyDietCoach -Weight Loss \\
\hline
\end{tabular}

ideal has similarly been shown promote unhealthy eating behaviors $[12,51,61]$.

\subsection{Research Activity 1: Preliminary App Review and Pilot Interviews}

To understand food journaling apps outside the Western context, we first sought to compare the common features used in Chinese and Western food journaling apps. We searched for apps from both cultural contexts in the Apple App Store, as the Google App store cannot be currently accessed in China. We selected apps based on keywords and number of reviews, modeling our search strategy after prior work systematically reviewing popular diet tracking apps [27]. We regarded any apps that focused on collecting and logging food information as food journaling apps, regardless of whether its goal was to build a healthier diet, manage weight, or some other goals. We scanned the top 200 free health \& fitness apps in both the Chinese and U.S. App stores and searched for relevant keywords in both Mandarin and English: food (饮食/食物), track (追踪), record (记录), dieting (节食), lose weight (减肥), health (健康), nutrition (营养), and calories (热量/卡路里). From these searches, we first identified 22 relevant Chinese food journaling apps and 22 relevant Western food journaling apps. We then removed all Chinese apps with fewer than 1,000 ratings and Western apps with fewer than 7,500 ratings, choosing these criteria as reasonable cutoffs between frequently and sparsely-reviewed apps in each marketplace. We note that Western apps were substantially more reviewed, on average, than Chinese apps. We finally reviewed 9 Chinese apps and 11 Western apps (Table 1):

We first collected common features, both tracking-related features and persuasive features, in Chinese food journaling apps and found their counterparts in Western apps, using simple descriptive analysis to count the frequency of features and taking screenshots of each feature, to compare how they were designed. Through this comparison, we realized that some persuasive features of Chinese apps heavily emphasized body image, while Western apps tended not to, which informed our later analysis. Since we later conducted a more formal analysis that specifically focused on persuasive features about body image (research activity 3 ), the results in Persuasive Feature Analysis Findings contain the main findings of this analysis, such as the frequency of features (Table 4) and their screenshots. The auxiliary material of this paper includes the complete table of this analysis of both tracking-related features and persuasive features.

We then developed an interview protocol based preliminary app review findings. We asked participants about their experiences with food journaling technology and assessed perceptions of design features which frequently appeared in either or both of Chinese and Western apps such as manual journaling, feedback on progress, challenges, rewards, reminders, and built-in social platforms by sharing screenshots of each of these features. We conducted two pilot interviews, with both expressing strong motivations to journal their food to achieve a particular body shape rather than diet, aligning with our preliminary app review. Since the focus on body image was both surfaced in the preliminary app review and the pilot interviews, we iterated our interview protocol to focus on understanding participants' perceptions of features that emphasize body shape and asking questions to understand the influence of cultural perceptions of beauty on people's experience with food journaling technology.

\subsection{Research Activity 2: Formal Interviews}

We then conducted semi-structured interviews to understand people's experiences of using Chinese food journaling apps and how they perceived features related to body image. Example questions included: How do you use food journaling apps? How do you feel about a particular food journaling app feature (e.g. success stories, an attractive model, the internal social platform)? Has your food journaling app influenced your perception toward body image or what a healthy diet should be? Do you have any personal experience related to body image?

We selected a few interfaces where Chinese and Western apps tended to differ in how they were designed for participants to react to in a card sorting activity. We focused the activity on features where Chinese and Western apps differed substantially, examining a subset of the persuasive principles surfaced in our feature analysis. We asked participants to sort interfaces showing builtin social platforms and containing specific elements relating to body image (before \& after pictures, user-posted body pictures, real person models, and cartoon characters). To keep the study manageable, participants reacted to 10 interfaces (4 interfaces of social platforms and 6 interfaces relating to body image), grouping them into "like/dislike/neutral" and explaining their categorization. For participants who could not read English, the researcher explained what the screenshots of Western apps were highlighting.

Eligible participants were Chinese adults (18 years and older) who had experience using a Chinese food journaling app, whether current or previous use. We recruited all participants (Table 2) online, posting our recruitment message on the first author's WeChat and Weibo's personal timeline, in WeChat and TenCent QQ's public chat groups, and to Weibo's public channels about weight loss or 
Table 2: Demographic information about participants

\begin{tabular}{ll}
\hline Demographic & Participants (18 total) \\
\hline Gender & 11 Female, 7 Male \\
Age & Min 20, Max 31, Mean 24.83, SD 3.22 \\
Profession & 4 Undergraduate students, 5 Graduate students, 1 Research assistant, 1 Content operator, \\
& 1 Salesperson, 1 Scriptwriter, 1 Product Manager, 1 Artist, 1 Government Employee, \\
& 1 Insurance Clerk, 1 Telecommunications \\
Chinese food journaling app used & 10 Mint, 4 iCalorie, 3 Food Storehouse, 1 Good Body Knowledge \\
How long they had journaled & 41-2 months, 6 3-6 months, 3 6 months-1 year, 3 2-3 years, 14 years, 15 years \\
Motivation for journaling food & 13 lose weight, 5 facilitate bodybuilding \\
\hline
\end{tabular}

food journaling. We also directly messaged Weibo users who mentioned the name of a popular Chinese food journaling app in a post they made and invited users who posted frequently on food journaling apps' internal social platforms. Two participants (P16m and $\mathrm{P} 17 \mathrm{~m}$ ) were recommended by $\mathrm{P} 13 \mathrm{~m}$ after the interview. All participants lived in China during the time of the study. Interviews were about 90 minutes on average. After the interview, we compensated participants with $140 ¥($ about $\$ 20)$.

We conducted the interview synchronously in Mandarin online through WeChat, a popular instant messaging app in China. The researcher sent questions in text, and participants responded with voice memos. We chose this mode because the interview involved sending screenshots of food journaling app features. Our two pilot studies compared this method against video calling participants, and we found that participants found it more convenient to respond in voice memos. A few participants occasionally chose to type their responses during the interview.

\subsection{Research Activity 3: Analysis of Persuasive Features in Apps}

Since many of the features that we examined during the interview were aimed to persuade people of the importance of having a perfect body shape, we systematically analyzed the design features in Chinese food journaling apps that emphasized body image, including some features participants sorted during the interview. We used Oinas-Kukkonen and Harjumaa's framework for persuasive systems design [58] to investigate what persuasive principles these features leveraged and how the use of persuasive features differed between Chinese and Western apps. We selected this framework because it has been widely-adopted for articulating persuasive design choices, including prior HCI studies [33, 59]. We first labelled features that emphasized body shape in Chinese apps based on persuasive principles listed in this framework. To compare the cultural difference, we then found corresponding features in Western apps to show how these persuasive strategies were implemented differently in Western culture.

There are four categories in the framework for persuasive systems design [58]: primary task support, dialogue, system credibility, and social support (Table 3). The primary task support category suggests that designs should leverage principles such as reduction, self-monitoring, and simulation, to help people more easily perform their task and reach their goal. The dialogue category suggests that designs should aim to motivate people to perform tasks by offering feedback such as verbal information, rewards, and reminders. Design principles in the system credibility category aim to make people feel that the system is credible by building trust via expertise, real-world feel, and authority. The social support category suggests that designs can motivate people by leveraging social influence through various social interactions such as social comparison, social learning, and recognition.

We found that Chinese food journaling apps leveraged 5 major persuasive principles to portray the thin ideal, which fell into 3 major categories: primary task support, dialogue, and social support. These 5 principles are reduction (the primary task support category), simulation (primary task support), similarity (dialogue), social comparison (social support), and recognition (social support). We did not find persuasive principles in the system credibility category that emphasize body image, since this principle tends to focus on increasing users' trust in the system.

\subsection{Data Analysis}

We analyzed the interviews using thematic analysis [9] through a mix of deductive and inductive approaches. Considering our intention to understand people's perception towards persuasive features that emphasize body image and the related cultural beauty norm, our codebook correspondingly contained 3 parent codes: positive perceptions, negative perceptions, and cultural influence, categorizing participant quotes based on these 3 themes. We then used the affinity diagramming to group quotes into different subgroups, resulting in 9 child codes: inspiration, move beyond food, instructive, anxiety towards the body shape, social anxiety, incorrect or harmful perceptions toward body image and weight-loss methods, unreal, Western culture, and Chinese culture. All authors met weekly throughout the coding process to discuss the codebook and findings. After coding, the first author then translated related quotes into English. We quote our participants as PXX with $\mathrm{f}$ or $\mathrm{m}$ indicates the participant's gender.

To analyze the app reviews, the first author, who is fluent in Mandarin and English, downloaded the selected Chinese and Western apps (Table 1) and open coded common features by examining every main page within the app and performing common food journaling tasks (setting a goal, journaling a food, examining goal progress, sharing food to the internal social platform). When noticing instances where Chinese apps suggested an ideal body image, the first author used the framework for persuasive systems design [58] to code the feature. From the list of persuasive principles utilized 
Table 3: Main categories in Oinas-Kukkonen and Harjumaa's framework for persuasive systems design [58]

\begin{tabular}{ll}
\hline Category & Principles \\
\hline Primary Task Support & Reduction; Tunneling; Tailoring; Personalization; Self-monitoring; Simulation; Rehearsal \\
Dialogue & Praise; Rewards; Reminders; Suggestion; Similarity; Liking; Social role \\
System Credibility & Trustworthiness; Expertise; Surface credibility; Real-world feel; Authority; Third-party \\
& endorsements; Verifiability \\
Social Support & Social facilitation; Social comparison; Normative influence; Social learning; Cooperation; \\
& Competition; Recognition \\
\hline
\end{tabular}

Table 4: Persuasive design principles employed in food journaling apps that relate to body image. The denominator of Western apps differs slightly between features because one Western app ( $W W /$ Weight Watchers) included a few features behind a paywall, and about half of the apps included an internal social platform

\begin{tabular}{llll}
\hline $\begin{array}{l}\text { Persuasive Design } \\
\text { Principle(Major Category) }\end{array}$ & Specific Feature in the App & $\begin{array}{c}\text { Prevalence in } \\
\text { Chinese Apps }\end{array}$ & $\begin{array}{c}\text { Prevalence in } \\
\text { Western apps }\end{array}$ \\
\hline Reduction & Body Assessment & $7 / 9$ & $2 / 11$ \\
(Primary Task Support) & Goal Setting: Allow to set an ideal goal & $7 / 9$ & $9 / 10$ \\
& Goal Setting: Warn of unhealthy weight goal & $5 / 9$ & $3 / 10$ \\
& Goal Setting: Allow to set a weight-loss speed & $4 / 9$ & $8 / 10$ \\
& Goal Setting: Warn of unhealthy weight-loss speed & $4 / 9$ & $8 / 10$ \\
Simulation & Goal Setting: Suggest the daily intake calories & $7 / 9$ & $9 / 10$ \\
(Primary Task Support) & Attractive Model: Real person model & $9 / 9$ & $2 / 10$ \\
& versus Cartoon figure & $6 / 9$ & $1 / 10$ \\
& Real-life Examples: Success stories & $5 / 9$ & $0 / 11$ \\
Similarity (Dialogue) & Provocative Statement: Statement about & & $0 / 10$ \\
Social Comparison & the thin ideal in the app description & $3 / 9$ & $6 / 11$ \\
(Social Support) & Unpleasant Experience & $9 / 9$ & \\
Recognition (Social Support) & Built-in social platform & & $0 / 6$ \\
\hline
\end{tabular}

in Chinese food journaling apps, the first author then examined whether or how the Western apps implemented similar features.

\section{FINDINGS: PERSUASIVE FEATURE ANALYSIS}

Chinese and Western food journaling apps tended to utilize different persuasive techniques to support people in achieving their journaling goals (Table 4). Though some Chinese apps contain persuasive principles that help people combat unhealthy journaling behavior, such as warning of unhealthy weight goals, Chinese apps were more likely to leverage some persuasive strategies to portray a perfect body shape. Western apps often used persuasive principles to aim to help people pursue healthy habits, and did not use some principles at all.

In the following sections, we follow the framework for persuasive systems design [58] to explain how these persuasive design principles are employed by presenting and comparing instances of features from commercial Chinese and Western food journaling apps. We translate the relevant parts of Chinese apps' screenshots. To protect poster anonymity, we blur out faces of users of the apps (e.g., people who post photos) and usernames in the screenshots.

\subsection{Primary Task Support}

The primary task support category of the framework for persuasive systems design aims to persuade people to continue using a system by reducing the effort needed to reach specific goals. Although food journaling can be used to promote general awareness of eating habits and management of chronic conditions, the commercial apps we analyzed (both Chinese and Western) primarily align with the frequent journaling goal of managing weight $[18,40]$. Food journaling apps tend to use two persuasive strategies to support the goal of managing weight, reducing the complex task of losing weight into smaller tasks and simulating the relationship between eating healthy or reducing calories to the desired outcome of losing weight and getting thinner.

4.1.1 Reduction. According to the framework for persuasive systems design, the reduction principle helps people more successfully perform a task by reducing a complex and difficult behavior or goal into simple and small tasks [58], aligning with the idea of developing incremental habits [31]. Food journaling apps usually leverage features such as goal setting and body assessment to help people begin their weight loss journey.

Regulation of Goal Setting 


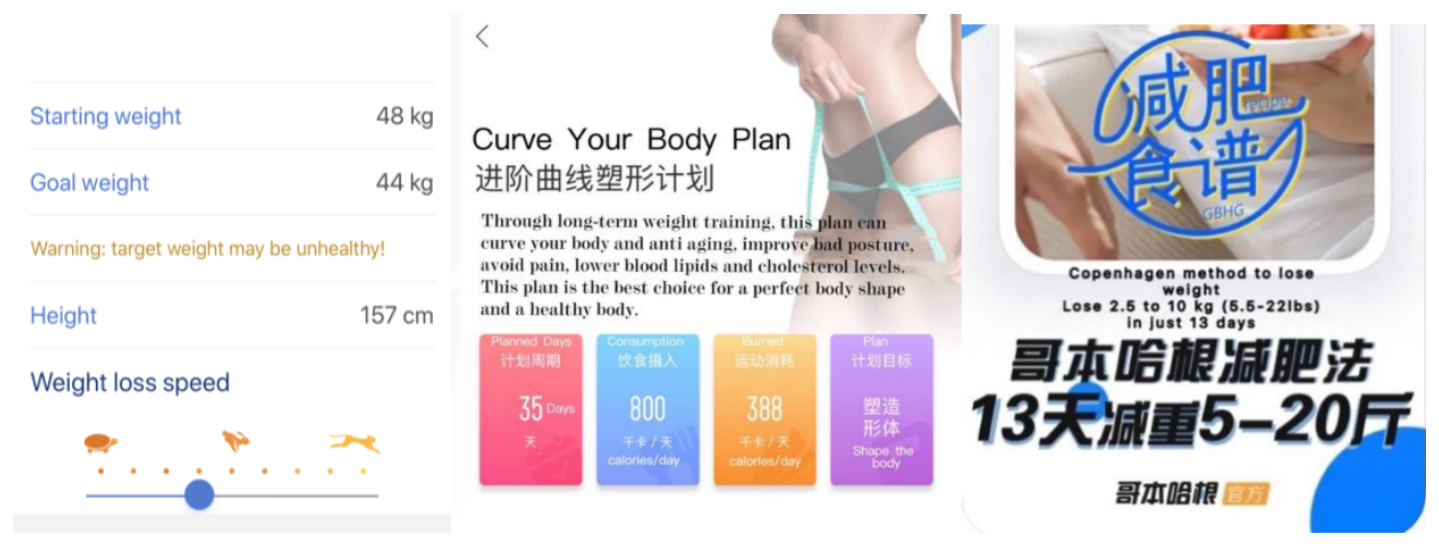

Figure 1: Weight loss speed indications in goal setting. (Left) the Western app Noom notifies users when they set an unhealthy weight loss goal. (Middle) the Chinese app Light+ provides an unhealthy weight loss plan. (Right) the Chinese app Copenhagen Method to Lose Weight supports unhealthy weight loss habits.

Though Chinese and Western apps leveraged similar strategies for goal setting, such as allowing a person to set a target weight (Chinese: 7/9; Western: 9/10) and suggesting daily consumed calories (Chinese: 7/9; Western: 9/10), more Chinese apps allowed a person to pursue an unhealthy weight goal by not regulating the weight loss speed through a target date or weekly rate of change (Chinese: 4/9; Western: 8/10). Some even promoted the goal of losing weight rapidly.

Public health authorities such as the U.S. and Chinese CDC recommend weight loss goals of no more than 0.5 to $1.0 \mathrm{~kg}$ (1 to $2 \mathrm{lbs})$ per week [47, 56]. Most Western apps (8/10) regulated the weight loss speed a person could set in the app to align with these guidelines by including text recommendations of healthy speeds or restricting the speed value to fall into a healthy range (Figure $1 \mathrm{Left}$ ) when setting the goal weight. However, fewer Chinese apps (4/9) supported setting and regulating weight loss speed. Other Chinese apps (5/9) promoted unhealthy diet plans that aim to help users lose weight rapidly. For example, the app Light+ (Figure 1 Middle) recommended a 35-day plan to only consume 800 calories, together with exercise to burn 388 calories per day. Public health guidelines suggest a minimum of 1,200 daily calories to ensure health [11]. Similarly, the app Copenhagen Method to Lose Weight (Figure 1 Right) promoted itself by claiming that users can lose 2.5 to $10 \mathrm{~kg}$ (5.5-22 lbs) in just 13 days, well more than the recommendations from public health authorities.

\section{Level of Details in Body Assessment}

Some apps include body assessment features to calculate measures associated with overall health or wellbeing, such as Body Mass Index (BMI), Basal Metabolic Rate (BMR), and body fat percentage. We found that Chinese apps (7/9) typically included a body assessment feature, while the fewer Western apps (2/9) which did only calculate BMI.

While assessing BMI and BMR can help people develop a better self-understanding of healthy habits when initiating a goal, among Chinese apps with body assessment features, two apps would classify someone with a normal BMI as "obese". Apps also lacked transparency around how such body fat percentage measure was calculated. For example, entering the height as $157 \mathrm{~cm}(5.15 \mathrm{ft})$ and the weight as $48 \mathrm{~kg}$ (105.8lbs), the Chinese app Weight Loss Secretary calculated the body fat percentage as $24.6 \%$, showing an ideal body fat percentage between $17-24 \%$. This app (Figure 2 Middle) further suggested $47 \mathrm{~kg}$ (103.6lbs) as the user's ideal body weight, suggesting an ideal bust of $83 \mathrm{~cm}$ (32.6 in), waist of $58 \mathrm{~cm}(22.8$ in), and hip as $85 \mathrm{~cm}$ (33.5 in). With the same height and weight, the Chinese app Change (Figure 2 Left) calculated the BMI as 19.5, falling into the normal range. However, the app assessed the body fat percentage as $23.3 \%$, annotating it as a "relatively high" body fat and rating the person as "mildly obese". Body fat percentage requires measurements beyond height and weight to accurately measure (e.g., whole-body air displacement plethysmography and dual energy X-ray absorptiometry [8]). We did not observe apps explaining how they estimated body fat percentage.

Compared with Chinese apps' emphasis on body assessment, only two out of nine Western apps calculated and reported BMI and none assessed other measures. These apps typically described BMI ranges, but stopped short of assessing a person's BMI (Figure 2 Right).

Taken together, the goal setting and body assessment features in Chinese apps may persuade people to pursue the thin ideal by first indicating the necessity for the person to lose weight and then providing unhealthy plans to reach the goal. The goal setting feature would assess people with normal BMI as "obese" without explanation or suggest how people's body should look like, making them to pursue unhealthy body shape goals or focus too much on their body shapes. Following the goal of achieving an ideal body shape, Chinese apps then provided unhealthy weight loss plans. These plans may also make people have unrealistic perceptions toward weight loss, believing that they could reach their ideal body shape goals in a short time.

4.1.2 Simulation. The simulation principle suggests that technology can leverage predictions or aim to see into the future to make people realize the longer-term relationship between their behavior and potential outcomes [58]. Chinese food journaling apps used three methods to simulate what life a person might experience once 


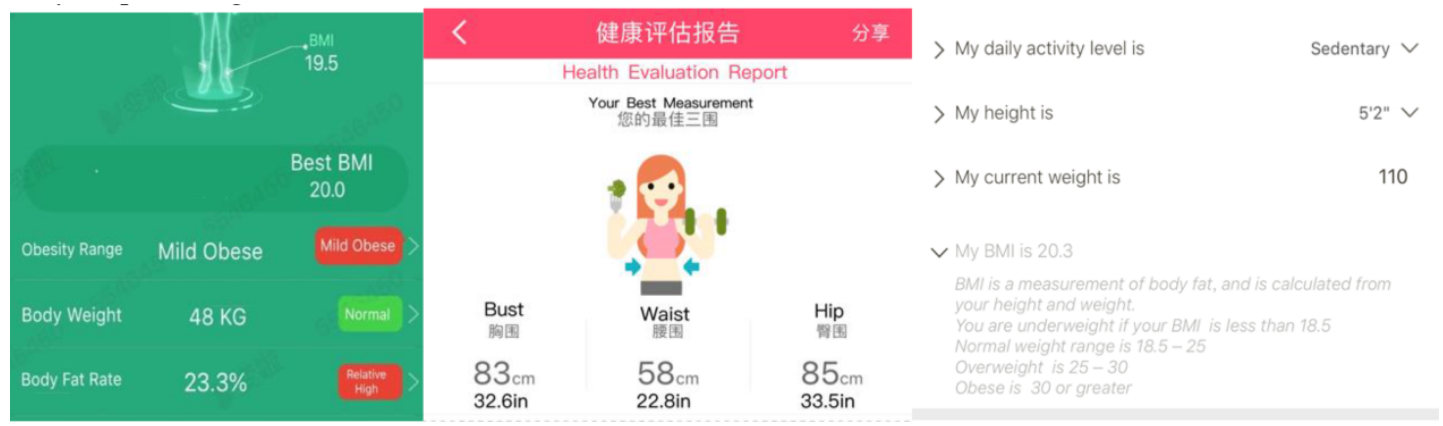

Figure 2: Body assessment feature of Chinese and Western apps for a user $157 \mathrm{~cm}(5.15 \mathrm{ft})$ tall and weighing $48 \mathrm{~kg}(105.8 \mathrm{lbs})$. (Left) The Chinese app Change, indicating a person as mildly obese despite a normal body weight. (Middle) the Chinese app Weight Loss Secretary, suggesting best measures for the user. (Right) the Western app Fooducate, calculating a user's BMI as 20.3 and providing an explanation for different BMI ranges.

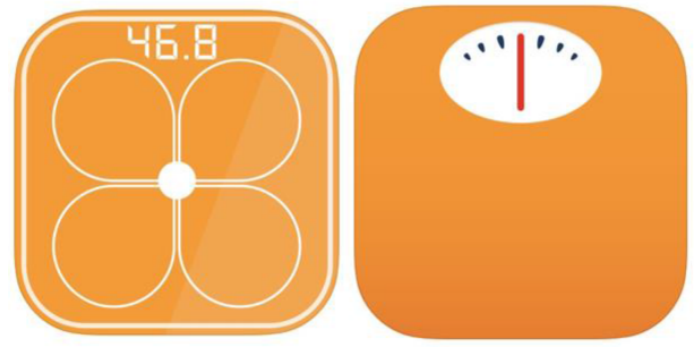

Figure 3: Apps use icons to convey messages about ideal or desirable properties. (Left) The icon of the Chinese app Good Body Knowledge shows a scale with the number " 46.8 $\mathrm{kg}$ (103lbs)", while (Right) the icon of the Western app Lose It! shows a scale without a number.

they lose weight: including visual hints or provocative statements which emphasize the importance of being thin, providing before \& after examples which highlight successful weight loss using the app, showing pictures of attractive models to signal life after successful weight loss. By comparison, Western food journaling apps rarely include simulations.

\section{Different Visual Hints and Provocative Statements}

Compared with Western apps, Chinese apps often used graphic elements and descriptions to relate body weight with physical appearance and even interpersonal relationships. These texts and images would typically evoke the particular importance of being thin, particularly for female users of these apps. Two Chinese food journaling apps evoke this desire for thinness in their names, as So Light and Light+. The logo of the Chinese app Good Body Knowledge (Figure 3 Left), shows scale with the number " $46.8 \mathrm{~kg}$ (103lbs)", which would be classified as underweight for Chinese people whose height is $1.6 \mathrm{~m}\left(5^{\prime} 2^{\prime \prime}\right)$ or taller (normal BMI range in China: 18.5-23.9 [19]), while the average height of Chinese females is about $1.6 \mathrm{~m}$ (5’2”) [4]. One Western app, Lose It! (Figure 3 Right), similarly uses a scale in their logo, but does so with a more abstract representation that avoids showing a number.

Chinese food journaling apps often included provocative statements targeted at female users, highlighting how good a person's life could be if they were slim. App Store pages of 4/9 Chinese apps mentioned "beauty", "beautiful", or "goddess" in their app descriptions. For example, Mint describes that "[our app has a] good weight loss community to witness your transformation to become beautiful [55]". The Change app touts that it "has helped 600,000 people to regain health and beauty. . making you become more beautiful [13]", while Weight Loss Secretary states that someone may "No longer a dream to become a goddess! [73]".

The descriptions of Western apps typically focus on how the app could facilitate establishing a healthier diet through scientific weight loss methods. We did not observe "beauty", "beautiful", "goddess" in any descriptions of Western apps. Instead, Western apps tend to promote how they can help achieve health outcomes, such as MyFitnessPal's description stating "Whether you want to lose weight, tone up, get healthy, change your habits, or start a new diet, you'll love MyFitnessPal [57]”. Similarly, for Lose It!, it described itself as "a calorie counting app that helps you reach your weight loss goal. Simply download the app, set your goals, and track your foods and exercises to lose weight [46]".

\section{Frequency of Success Stories}

Most Chinese apps (6/9) we examined prominently included stories of users who successfully lost weight to inspire other users, whereas one Western app included this feature. In addition to showing pictures of users before \& after their weight loss, apps would also describe how attractive or successful the person had become after successfully losing weight.

For example, the Change app (Figure 4 Left) presented successful examples by indicating the positive effects of losing weight and having a thinner body figure, such as "Lost $32.5 \mathrm{~kg}$ (72lbs) in 7 months. [It is] easy to be fat-Meet the most beautiful you at the best of times", using a picture of a person in a wedding dress to highlight the "after" effects. Similarly, the Mint app (Figure 4 Middle) emphasized benefits of being thin on its landing page, describing a person as upset in "before" and happy in "after" pictures: "Lost $11 \mathrm{~kg} / 24.2 \mathrm{lbs}$ in 6 months, [turning] from a fat girl into a smiley goddess". The only Western app (Carb Manager, Figure 4 Right) which provided stories highlighted the amount of weight the person had lost and showed before \& after pictures, but did not further discuss the personal or health outcomes of the weight loss. 


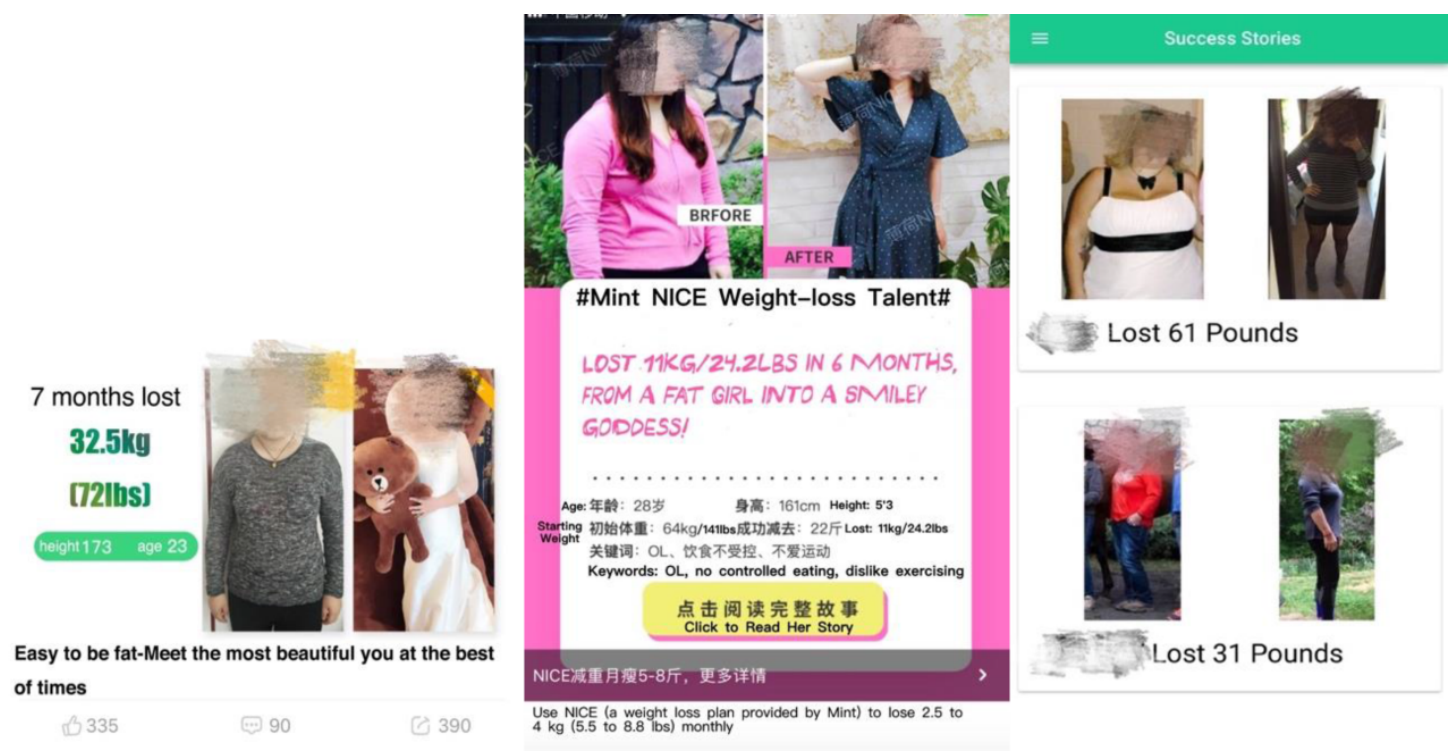

Figure 4: Success stories were more prevalent in Chinese apps than Western apps. (Left) The Chinese app Change and (Middle) Mint describe the positive effects of being slim, while (Right) the Western app Carb Manager only shows before \& after pictures and the weight loss amount.

\section{Use of Attractive Models}

All Chinese apps (9/9) included photos of models, mostly female, with a thin body figure in various interfaces, such as the landing page, in-app banners, icons of buttons, and pictures contained in articles or tutorials provided by the app. No Western apps contained pictures of models in these places.

For example, the Chinese app Weight Loss Secretary put models on its landing page and homepage (Figure 5 Upper Left and Upper Middle) to call for people's participation in weight loss challenges. These models could also show how useful the app and its challenges could help attain an ideal body shape. Another app Change (Figure 5 Bottom Left) put models on its eCommerce platform with statements, such as "be thin be beautiful", to imply that people would become as beautiful as the displayed models once they use their weight loss pills or nutritional meals. Similarly, the app Mint (Figure 5 Bottom Middle) used the picture of a model measuring her thin waist as the thumbnail of a recommended weight loss video tutorial, visualizing the outcome of having a satisfying body shape once using the app. Western apps seldomly contained pictures of people in their interface, except pictures shared by users on the built-in social platforms. Two Western apps leveraged cartoon characters to include pictures of successful weight loss. For example, MyDietCoach (Figure 5 Upper Right) Western apps used cartoon figures as used a virtual avatar to show what a person might look like by making the left avatar resemble the user's current body figure, and the right avatar represent the user's ideal body figure. In the app's premium version, the left avatar became slimmer as the person loses weight.

All together, these simulation features strongly emphasized the cultural beauty norm and body image by using textual and visual attractions, such as provocative statements that linked positive effects with thinness and attractive models whose body shapes aligned with the thin ideal. These features aimed to persuade people that a perfect body shape should be thin. To further persuade people to pursue thinness, they emphasized the benefits of losing weight and being thin. Particularly, the graphic elements, such as the model and the success stories, could explicitly show how beautiful or attractive people can become as long as they use the app and even buy the app's products.

\subsection{Dialogue}

The dialogue category intends to persuade users through various interactive feedback to keep a person moving towards their goal, such as by offering praise or reminders. Chinese food journaling apps tended to use the similarity principle in this category to persuade users to have a "perfect" body shape by relating weight to negative life events or outcomes.

4.2.1 Similarity. The similarity principle aims to offer feedback that makes users think of themselves by reflecting or reminding them behaviors or situations they have faced [58]. Chinese food journaling apps (3/9) practiced this principle by prompting people to think about their personal experiences of feeling "fat" through graphic elements or social features. We did not observe Western apps utilizing this principle.

Use of Unpleasant Similar Experiences to Trigger People

A few Chinese apps highlighted the challenges an overweight person might face. Apps leveraged the similarly strategy by presenting overweight models or highlighting depressing moments brought on by being overweight, aiming to resonate with people's personal experiences or thoughts. This differed from how apps leveraged the simulation principle, where apps instead used pictures of models to remind a person of what could be if they successfully lost weight. 


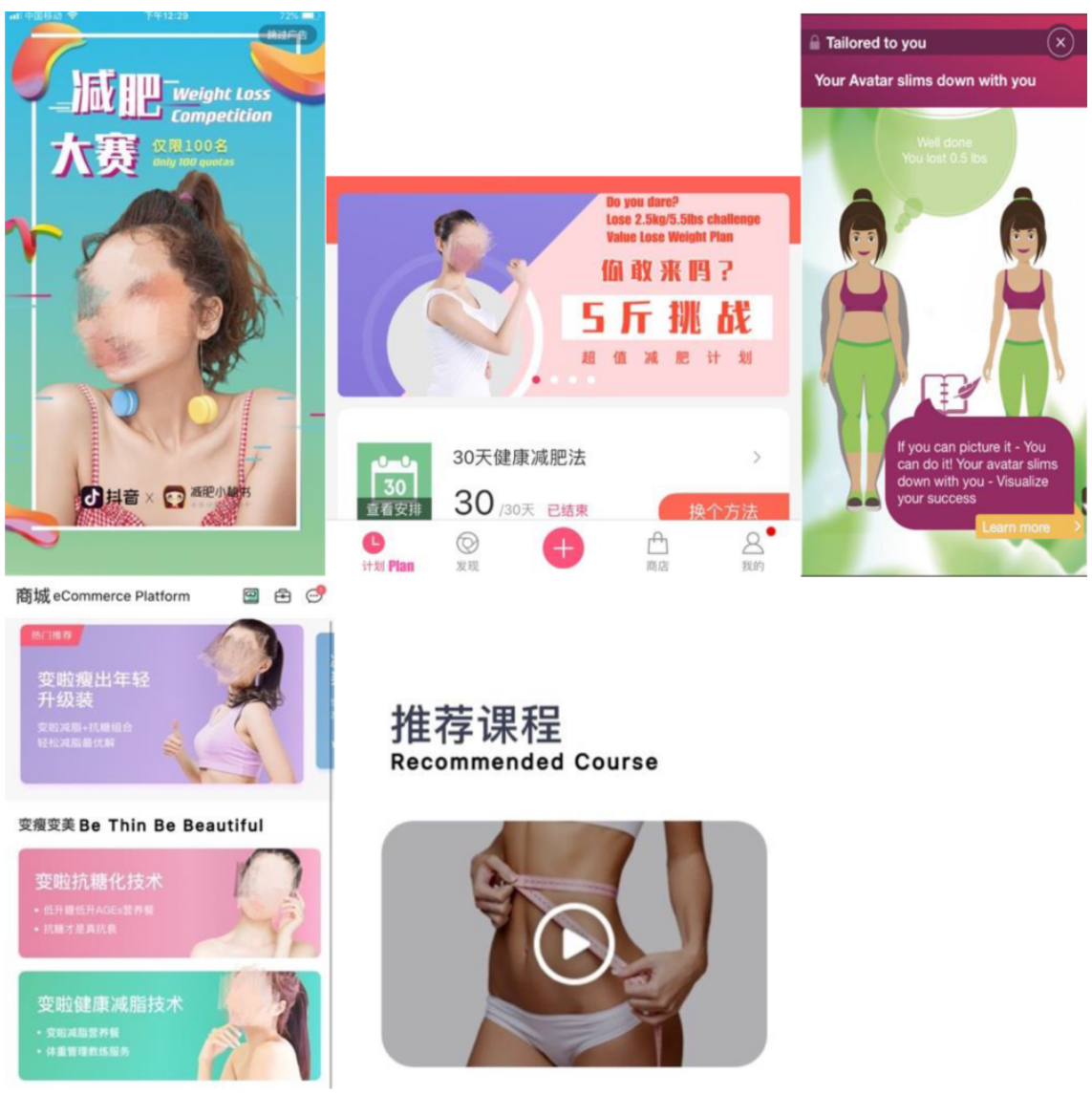

Figure 5: Chinese apps often used attractive models in many places to evoke a thin ideal, such as in Weight Loss Secretary (Upper Left; Upper Middle), Change (Bottom Left), and Mint (Bottom Middle). No Western apps included models, but a few like MyDietCoach (Upper Right) used cartoon figures to evoke this ideal.

For example, the App Change (Figure 6 Left) uses images of unhappy and overweight models in its interface to describe the downsides of their life: "Life of people who are overweight: lack of confidence, fear to eat, fear to wear". Another app, Weight Loss Secretary (Figure 6 Right), has a subsection in its internal social platform named "Overweight Roast" to let users complain about their negative experiences because of being "fat" (at least, the users who felt they were fat).

Chinese apps leveraged this kind of unpleasant body image experience to motivate users to lose weight. For example, in the Weight Loss Secretary's "Overweight Roast" section, one user complained about her negative experience of gaining weight during pregnancy, and she encouraged other users to lose weight: "I gained $25 \mathrm{~kg}$ (55lbs) in my pregnancy and I felt people all looked at me strangely! Though I knew my husband did not give me the cold-shoulder and always treated me well, I know men always like women with good body shapes! So girls, do not believe people saying you are not fat and you look good. You will only know how beautiful you are when you are thin".

This similarity principle aimed to persuade people to pursue thinness by recalling and reinforcing the negative aspects of being "overweight". Either through the content created by the app itself or struggling experiences shared by other users, people would be motivated to lose weight as they may want to avoid facing these painful moments.

\subsection{Social Support}

The social support category was often seen in the app's social features, which leverage social influence to persuade users to perform a behavior [58]. Compared with Western apps, Chinese food journaling apps leveraged social features more often, such as having built-in social platforms (Chinese: 9/9; Western: 6/11), allowing people to invite their friends to use the app with them (Chinese: 5/9; Western: $1 / 10$ ), and supporting sharing food logs to other social platforms (Chinese: 9/9; Western: 3/10). Chinese apps mainly applied social comparison and recognition to emphasize body image, such as appreciating perfect body shapes or promoting the thin ideal.

4.3.1 Social Comparison. The social comparison principle aims to persuade people to perform a target behavior by comparing their performance against others [58]. Chinese apps included social features for sharing pictures of body shape and weight loss progress, 

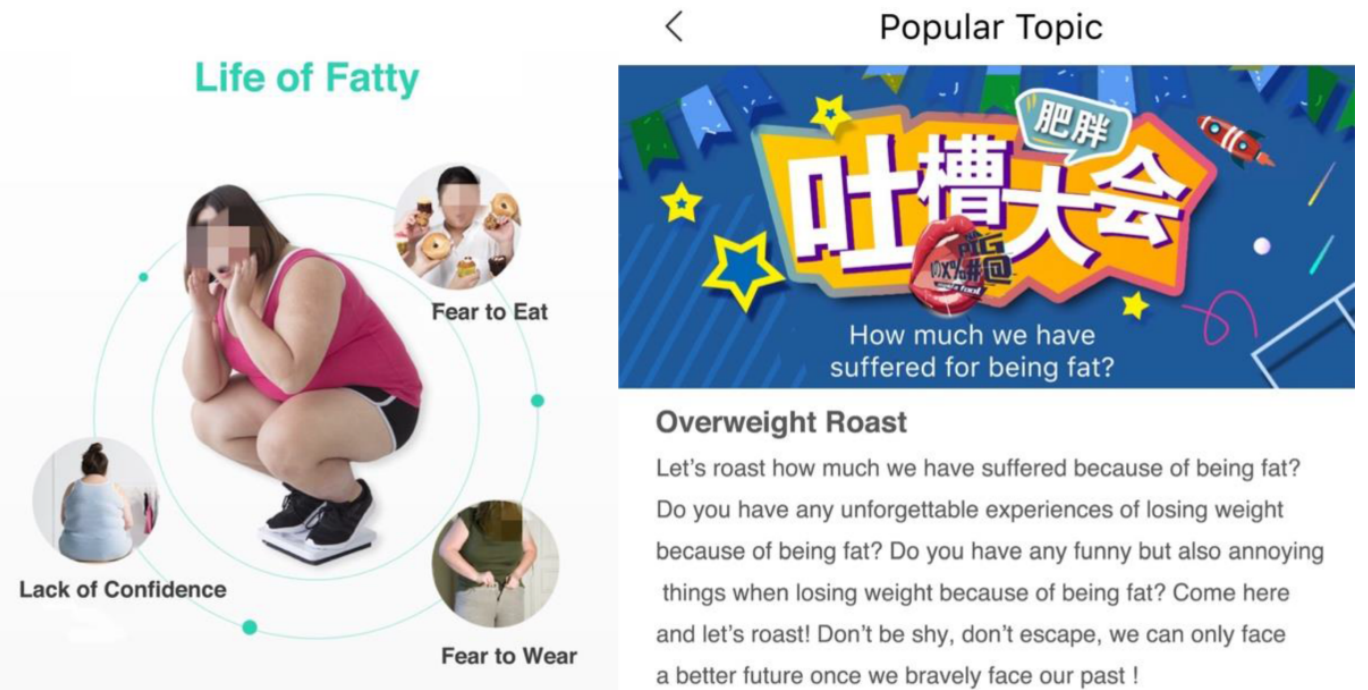

\section{Overweight Roast}

Let's roast how much we have suffered because of being fat? Do you have any unforgettable experiences of losing weight because of being fat? Do you have any funny but also annoying things when losing weight because of being fat? Come here and let's roast! Don't be shy, don't escape, we can only face a better future once we bravely face our past !

Figure 6: A few Chinese apps highlighted the unpleasant experience of being overweight, such as by describing the life of someone who is overweight in the App Change (Left) or giving people a space to share unpleasant experiences in the Weight Loss Secretary (Right).
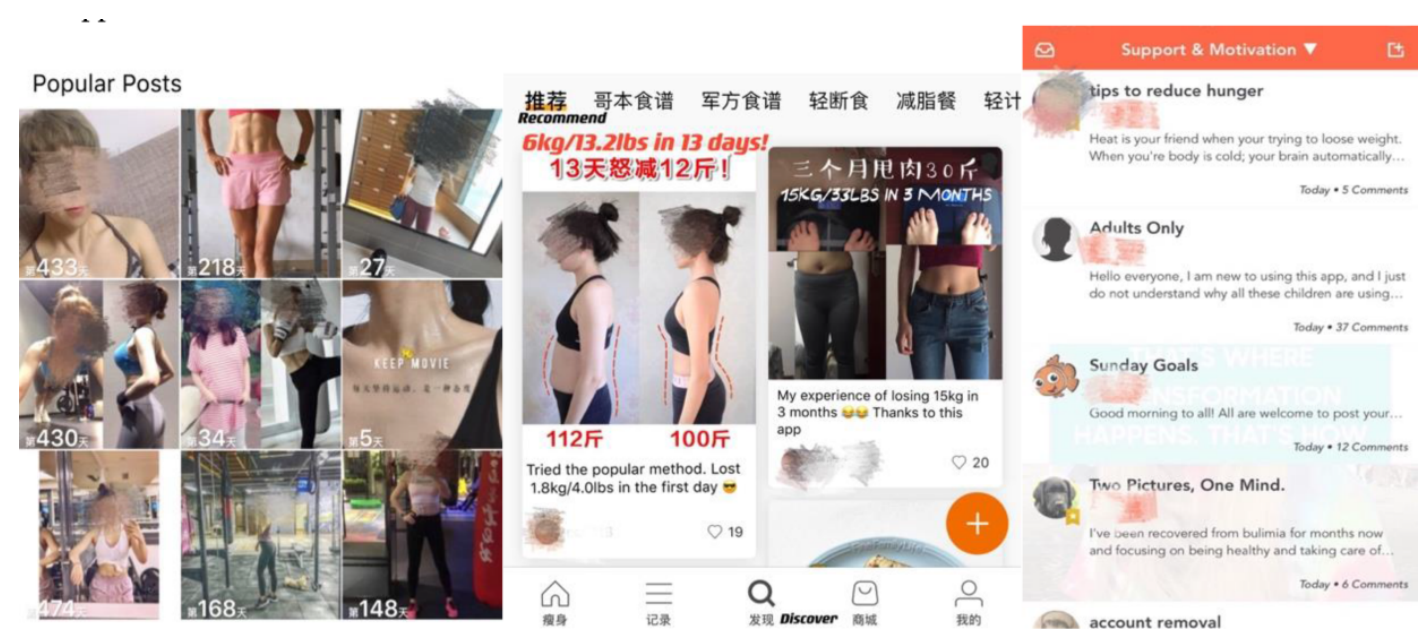

Figure 7: (Left) the Chinese apps So Light and (Middle) the Copenhagen Method to Lose Weight focus on presenting pictures of people's fitness and weight loss journeys through pictures on the internal social platform, while the Western app My Plate (Right) emphasizes text posts rather than their pictures.

while Western apps did not support this comparison as readily because social platforms were primarily text-based.

The layout of Chinese apps' built-in social platforms (Figure 7 Left and Middle) typically resembled that of Instagram or Pinterest, showing images and enabling viewing a person's post history. Western apps' built-in social platforms tended to be modeled after Q\&A forums, emphasizing text posts and avatars rather than pictures from users of the apps (Figure 7 Right). The Chinese app So Light's social platform highlighted pictures from popular accounts in a social feed (Figure 7 Left), while the Western app My Plate's internal social platform (Figure 7 Right), emphasized text content.
My Plate did support sharing user photos, but in the main forum these pictures showed up as the background of text posts rather than being highly emphasized. Another Chinese app Copenhagen Method to Lose Weight (Figure 7 Middle) emphasized both the pictures of people's body shapes and the text, such as "[I] tried the popular [weight loss] method [online]. [I] lost $1.8 \mathrm{~kg} / 4.0 \mathrm{lbs}$ in the first day", to show that people could easily get an ideal body shape by using the app.

4.3.2 Recognition. The recognition principle aims to make someone perform a target behavior by offering public recognition of 


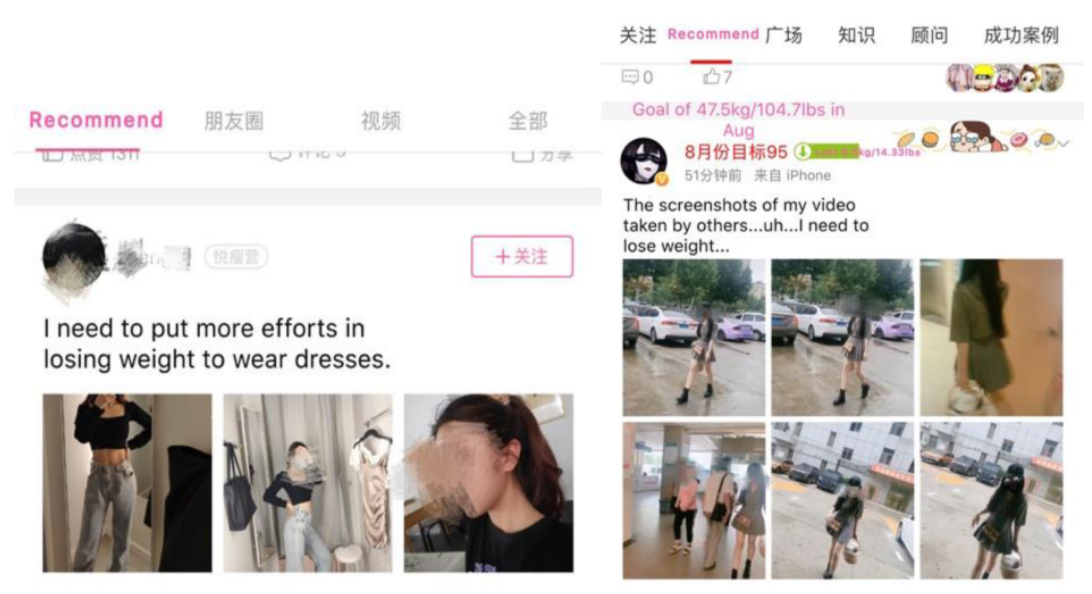

Figure 8: The recommended posts on the homepage of the built-in social platform of Chinese apps often highlighted users who expressed needing to lose weight, including in Light+ (Left) and Weight loss Secretary (Right).

the successes [58]. Chinese food journaling apps employed this principle by selecting and recommending posts from other users on the homepage of the built-in social platform (7/9). However, the social platforms of Western apps did not utilize this strategy $(0 / 6)$, sorting posts either by popularity or recency.

The built-in social platforms of Chinese apps (Figure 8) often recommended posts of female users who were already thin, but still expressed the need to lose weight. For example, the Chinese app Light+ (Figure 8 Left) recommended a post on its social platform's homepage where the user claimed that she needed to put more effort in losing weight to wear dresses, while showing her abs in the picture. Similarly, Weight Loss Secretary (Figure 8 Right) recommended posts in which the user expressed disappointment about her body shape when sharing her pictures: "[These are] screenshots of my video taken by others... uh...I need to lose weight". Chinese apps, such as the Copenhagen Method to Lose Weight (Figure 7 Middle), also offer recognition to people who use the app to lose a lot of weight in a short period of time, suggesting how useful the app was.

Overall, we found that Chinese apps used a variety of persuasive design principles to portray a "perfect" body to aim to achieve, while persuasive principles were primarily used to pursue health by managing weight in Western apps. Promoting a single-dimensional beauty norm around body image, Chinese apps aim to utilize these features to persuade people that they still do not have perfect body shapes, that they can easily achieve the perfect body shape just by using the app, and the real-world benefits of having a perfect body shape. For example, some Chinese apps offered public recognition to people who support the cultural beauty norm of thinness. Other people who saw these posts may have this cultural beauty norm reinforced, and correspondingly feel dissatisfied about their own body shape. In contrast, we find that Western apps rarely utilize persuasive features to emphasize body image, suggesting that persuasive approaches are highly influenced by cultural perceptions. Furthermore, the beauty norm about body image promoted by Chinese food journaling apps aligns with Chinese mainstream cultural norms around beauty.

\section{FINDINGS: INTERVIEW STUDY}

Participants' cultural norms influenced their perceptions of the persuasive strategies Chinese food journaling apps used to emphasize an ideal body shape. Participants who agreed with the cultural beauty norm adopted by Chinese food journaling apps felt the persuasive strategies were necessary, motivational, rich, and instructive. For participants who held a different cultural norm, they negatively felt about these persuasive strategies because they could trigger anxiety and proliferate inaccurate perceptions toward body image and weight loss.

\subsection{Chinese Food Journaling Apps Reflect Cultural Beauty Norms}

Some of our participants embraced Chinese apps' concentration on body image because they agreed with the cultural beauty norm that pursues the thin ideal. Aligned with the famous Chinese saying, “Beautiful girls shall never weigh over $50 \mathrm{~kg} / 110 \mathrm{lbs}$ (好女不过百)”, several female participants stressed their wish to be under $50 \mathrm{~kg}$ : "My weight and height are in normal range but you know the saying that girls shall never weigh over 50kg" (P4f) and "Girls will never think themselves thin enough, I think I will be prettier if I am 100 pounds"(P15f). Moreover, some participants felt that a good body shape was crucial for an individual's life success: "I feel a good body shape can positively influence your confidence and your job seeking or interview. I wish I can have a better body shape so that I will be confident wherever I go" (P15f). Some male participants in our study similarly commented on the expectation that females have a good body shape is a way to materialize females: "The subconscious in China culture is still about patriarchy that the female's appearance is part of their values. It somehow materializes females" (P10m).

Compared with Chinese apps' use of persuasive techniques to emphasize body image, participants noticed Western apps put health as the priority: "These foreign apps may focus more on health instead of pursuing good body shape. However, apps in China care more about the body shape. Especially for females, we pay attention to having slim waists and skinny legs. People always say that good body shape is to be thin" (P15f). Participants felt the reason why 
Western apps tended not to emphasize a perfect body shape was mainly a perception that Western culture has different or more diverse attitudes towards beauty: "You [meaning the interviewee] live in the foreign country and you know that foreign countries have a different attitude towards the body shape, which is different from the pursuit towards thinness in China" (P4f) and "It is emphasized in foreign cultures to not judge others' body shapes" (P7m). Additionally, participants felt Western apps focused on providing practical functions for managing weight and building healthy eating habits while Chinese apps might nudge users because they might be less experienced: "Users of Western food journaling apps may already have some basic knowledge about what they shall do to [reach their goals] or how to use the app. The Western-style apps emphasize functionality rather than the [Chinese apps'] overusing persuasive behaviors to make beginners quickly become their own users" (P10m).

However, our participants still agreed with the persuasive strategies used by Chinese apps to promote the importance of a good body shape, as they were accustomed to the Chinese beauty norm of a thin ideal: "I like that people in Western culture appreciate different body size because they all have their own beauty. However, for Chinese people, maybe it's more inspirational to emphasize the body image. If you want to have a healthy diet, you would use recipe apps. People definitely want to keep a good body shape and lose weight to use this app. Therefore, the app needs to have some pictures about the good body shape to motivate users, making them feel you are a professional app" (P9f).

\subsection{Chinese Apps and their Adopted Cultural Norm Support Users Who Share the Same Goal}

For participants who journaled their food to gain a better body shape, they regarded the persuasive features that emphasize body image as inspirational as they can simulate a satisfying outcome that a person could obtain by using the app. When seeing posts shared by other people on the internal social platform, P18f tended to be inspired by "girls with a similar background as mine but have a very good body shape". Participants also felt inspired by the success stories shared by the app or before \& after pictures posted by other users. For example, P1f felt a before \& after picture was "motivational because it enables me to 'see' my successful weight loss result". Similarly, P5f thought a before \& after picture was a strong motivator for her because "it can make you understand how goodlooking you will be after losing weight". Participants commented on the body shape of the images they saw in apps: "I like to see posts shared by good-looking people" (P18f) and "I like this interface because the model on this page is attractive" (P2f).

To better facilitate their goals related to body image, some participants believed that the content of food journaling apps should move beyond food. Since some people use food journaling apps in order to gain a better body shape, some participants felt that food journaling apps should contain persuasive features that emphasize body image: "[the app that allows] sharing pictures, such as people's abs and of course their dieting behaviors, align with people's expectation for this kind of app" (P17m). P18f believed: "I actually think it is good to add some elements about body image properly to motivate us. People would feel bored and less motivated to just see food pictures or social tracking about physical activities". As a comparison, some participants had less interest in Western apps, as they either focused on presenting information in text or used cartoon characters to emphasize body image instead of real people's photos. For example, the internal social platforms of Western food journaling apps tend to show the food that people eat, their weight-loss progresses, and methods for losing weight in words rather than pictures. Participants felt they were "boring" (P18f) because "It is all about words and the icons look 'serious'. Using the food journaling app is actually a 'serious' behavior and I feel (having pictures) can make it more relaxing" (P10m). Participants also felt cartoon characters were less "trustworthy" (P10m; P3f) because "the cartoon avatar can't represent people's body shape because everyone has a different body shape" (P3f).

Participants felt that persuasive features which emphasize body image could also be instructive for people whose goals are to gain a better body shape, as some features provided practical guidance for people who aim to achieve these goals. For example, many users of the apps posted detailed guides with pictures, even short videos, to educate others how to eat and exercise to lose weight. P9f felt about these posts: "It is real and normal people's experience. I feel you can learn something from it". Similarly for the success stories feature, people would introduce their weight loss progress, share how they overcame their struggles, and describe how they achieved their success. Since some people already felt pictures shared by other people were motivational, when these motivational pictures were combined with practical information, they may have a better instructional usage than tutorials or suggestions provided by the app. P10m felt, "In China, the food journaling apps' users mostly are new beginners or we can even say these people are just imagining to own a good body shape. However, they never follow weight-loss or strength training methods strictly. So these apps are using pictures to guide users".

\subsection{Chinese Apps and their Cultural Norms Can Exclude and Negatively Influence Some Users}

While some participants felt the persuasive strategies around body image included in Chinese food journaling apps can "attract and motivate" $(\mathrm{P} 13 \mathrm{~m})$ people, we found that this emphasis could also exclude some people when their journaling goal or cultural norms were not aligned with the cultural norms that the app promoted. Participants felt the way Chinese food journaling apps promoted a "perfect" and thin body shape may lead people to incorrect or unhealthy perceptions of losing weight or body image. The overemphasis on body shape in persuasive strategies could make people blindly pursue perfect body shape: "These apps are creating an unhealthy guide by making people only pursue muscles and abs rather than a healthy body. Healthy body shape does not have to build obvious muscles" (P7m). Additionally, participants felt success stories (e.g., Fig.7) could give people a false perception that weight loss is easy: "I do not like apps which promote it is easy to lose how many pounds" (P6f).

Participants felt that using persuasive strategies to emphasize body shape could also negatively influence people, including those whose goal or beauty norm aligned with the cultural norms that the 
apps were conveying. Some participants felt disappointed in their "imperfect" body shape, feeling that apps reinforces this notion: "I do not post my body pictures because I don't think my body shape is good. Also, seeing others' body pictures make me self-abased because they have abs but I have the muffin top" (P5f). Besides triggering anxiety towards body shape, participants felt that persuasive features that allow people to share their own body shape could also bring social anxiety. Participants felt the goal of sharing was primarily to socialize with others: "It is so obvious that their goal is for sociality. People share to let more people see and like their posts" (P15f). P1f felt similarly: "I use the platform to see people's experience, learning what you eat. I am not here to see you brag out your body". Contrarily, Western apps were less likely to raise people's negative emotions because Western apps prioritize information over sociality. Participants felt focusing on words rather than pictures could motivate people to exchange experiences: "It is clearly a Q\&A platform that people would ask for help and other people would provide answers. I think it's really a good interaction" (P8m). Participants thus felt Western apps were "not for showing off body figure" (P11f).

Moreover, participants did not trust some persuasive design principles, feeling that the photos or stories were "not real" (P15f) People feel some users would share pictures that are "photoshopped" (P10m). Participants also felt that apps' use of attractive models and success stories was to promote their own products, such as the meal replacement and fitness equipment: "It is like selling a product. I want to see normal, real, and good looking pictures" (P8m) and "It's to sell products and cheat you to get money" (P9f).

As a comparison, some participants felt Western apps designed features that incorporated body image in a more professional way. Compared with Chinese apps, Western apps rarely contained pictures of the real person's body figure. Besides posts shared by users themselves, one of the few places where Western apps utilized a picture of a real person was in a workout tutorial, which participants were generally positive about because "These pictures are used to guide people to do exercises. Also, the pictures of the coach look quite real [not photoshopped]" (P8m).

Overall, we find that Chinese apps' persuasive approach to emphasizing body image and portraying a perfect body shape can influence people differently depending on their reason for journaling and their alignment with the cultural norm the apps convey. For people whose goal of journaling food is to lose weight or whose beauty norm matches the single-dimensional beauty norm, apps could support people's behavior change processes well, and they typically felt positive about how the persuasive features conveyed body image. However, for people who do not hold similar goals or beauty norms, they felt discouraged and excluded from the app. Even for people with the same goal or beauty norms, the heavy emphasis on perfect body shapes in Chinese apps would sometimes influence them negatively, such as raising their anxiety towards their own body and how they fit into society.

\section{DISCUSSION}

Overall, we found Chinese food journaling apps leverage persuasive design principles to portray perfect body shape and promote the thin ideal, an ideology heavily influenced by cultural norms. Though the Western counterparts utilize some of the same persuasive design principles, their primary goal appears to be to help people become healthier through managing weight. Participants perceived Chinese apps' emphasis on body image differently based on their personal goals and internalized beauty norms. For people whose goal and beauty norm reflects the thin ideal, the app's emphasis on body image can instruct new users, motivate people to keep using the app and stick to their goals, and enrich the content of food journaling apps. However, for people who have a different goal or beauty norm, it could trigger people's anxiety towards their body and sociality, lack reliability, and cause wrong perceptions toward losing weight and body image. Interestingly, although participants felt positive about how the Western apps prioritized health, they still believed that it was necessary for Chinese apps to emphasize body image when their own perceptions toward body image aligned with the cultural beauty norm.

\subsection{The Cultural Norm of Beauty and Its Raised Inclusiveness Issues in Chinese Food Journaling Apps}

In this study, through app reviews and empirical interviews, we found that the cultural norm of beauty became a necessity in both the design of Chinese food journaling apps and people's in-situ tracking experiences. Technology is rarely designed in a neutral way that removes any cultural influences. How people read information, how designers design the interface, and what messages are conveyed by the system are inevitably influenced by designers' cultural norms. Our findings show that persuasive designs which closely follow cultural norms can lead to exclusion and problems even for people who live in that context.

Our findings lead us to first think and reflect on the reason why Chinese food journaling apps heavily incorporate body image into persuasive strategies while Western apps do not. Our study suggests that a single-dimensional cultural norms around beauty in China seem to be a major reason account for this focus [74]. Eastern Asian cultures tend to accept the thin ideal as a societal norm [42] while Western cultures have a more diverse beauty concept which could appreciate both the thin ideal and sexual maturity [74]. Therefore, compared with Western countries, Eastern Asian countries have faced a more severe situation brought by the thin ideal. A study compared body satisfaction among Chinese, Indo-Asians, and European participants, finding that Chinese participants scored the lowest [41]. A similar study found that Chinese students were more dissatisfied about their own body image and thereby having more dieting behaviors than their counterparts in the United States [20]. Especially for Chinese females, rather than losing weight to be healthy, body image and the pursuit towards beauty seem to be the major motivation that drives Chinese females to lose weight $[66,75]$. About $73.36 \%$ Chinese undergraduate female students have tried to lose weight in the past 6 months and $30 \%$ are already underweight [75]. Female's concerns about body image are mainly caused by the mainstream and single-dimensional cultural beauty norm in contemporary China that "the slimmer the better" $[43,48,76]$.

As a result, this single-dimensional cultural norm of beauty in China which pursues thinness and portrays perfect body shape deeply influences the design of food journaling apps. To align with culturally embedded body image goal, Chinese food journaling 
apps inevitably leverage persuasive designs to reinforce this cultural beauty norm and persuade people to get "perfect" body shapes through showing people that they are not thin enough, promoting the benefit of having "perfect" body shape, and providing instruction for people to have satisfying body shapes "easily". Meanwhile, persuasive design principles were used to help manage weight or build healthy eating habits in Western apps. Though prior studies $[21,23,37]$ suggest that the persuasive strategies utilized in Western food journaling apps may also promote unhealthy weight loss goals such as ED practices, popular Western apps tend to not center body image as directly and promote these practices as much as Chinese apps do. Therefore, considering the nudging intention of persuasive designs [63], persuasive features that cater to cultural beauty norms could create more convincing messages for people who have the same belief, pushing them to more thirst for and be more determined to pursue the thin ideal.

Though the cultural beauty norm seems to be a necessity when designing Chinese food journaling apps, we find that these culturally designed systems can be exclusive, discouraging people whose goal and beauty norms are in conflict with the one that the app promotes. The current single-dimensional cultural beauty norm promoted by Chinese apps only appreciates "perfect" body shape and thinness. As we discuss above, many features are correspondingly designed to support and even promote this beauty norm, such as offering public recognition to people who express wishes to be skinny. As a result, people using the app for an eatingrelated goal or people who have a different beauty norm would feel excluded by the app. Even for people whose goal reflected the single-dimensional cultural beauty norm, they could be negatively influenced as certain persuasive features could cause their anxiety and wrong perceptions toward body image.

Furthermore, the current Chinese apps seem to also exclude male users since these apps often emphasized the beauty norm targeted at females. For example, the number " $46.8 \mathrm{~kg}$ (103lbs)" on the logo of the Good Body Knowledge app is far less than the average weight of Chinese adult males $(69.6 \mathrm{~kg} / 153.4 \mathrm{lbs}$ [69]). Also, the simulation principle in Chinese apps mainly uses female models or words related to females to portray "perfect" body shapes.

\subsection{Tension between Cultural Beauty Norm and Domain Knowledge in Designing Chinese Food Journaling Apps}

Besides inclusiveness, we also notice a tension between the singledimensional cultural beauty norm and scientific domain knowledge of healthy diet and body weight. As a health \& wellness technology, it is necessary for food journaling apps to include and leverage professional and scientific domain knowledge to help people build healthier eating habits. However, the seemingly crucial scientific knowledge may be in conflict with the influential cultural norm of beauty. Cultural beauty norms may promote scientifically incorrect or dangerous ideology around health and wellness, and designing health and wellness apps to follow these cultural norms can potential lead to various negative consequences. For example, our feature analysis observed instances where a person with a nearly-underweight BMI by Chinese CDC standards (19.5) would be classified as overweight by some Chinese apps, while studies have found that being underweight had a higher mortality rate compared with normal weights [29]. Therefore, the empirical findings of this study pose a question for us as both HCI researchers and designers: should we design to aim to subvert cultural norms for the sake of people's health, or follow cultural norms to better support people's goals even if they are unrealistic and unhealthy?

When a design does not align with cultural norms, even if it could pose less negative influence on people, people who live in that socio-cultural context might still view it as less useful and have less interest in engaging with it. In our study, participants did appreciate how Western apps prioritize health and Western culture is more inclusive regarding various body shapes. However, they still felt it was a necessity for Chinese apps to emphasize body image as "People definitely want to keep a good body shape and lose weight to use this app" (P9f). Therefore, this makes it challenging to design technology which hopes to subvert cultural norms. Some prior HCI studies have explored designs that aim to subvert cultural norms, such as a mobile app that tried to change women's sociocultural understanding of their own vagina [3] and a software that leveraged cultural insights to overcome cultural taboos to teach students knowledge about HIV [68]. However, our findings suggest that people prefer designs which align with their cultural perception around what the technology should persuade them to do. As many of our participants were agreed with and influenced by the Chinese cultural norm of beauty, the Chinese food journaling apps which "persuade" people to pursue thinness seem to better support their goals than Western apps. Taken together, when designing Chinese food journaling apps, not subverting the cultural norm of beauty at all does not mean ignoring necessary scientific domain knowledge. Considering the negative influences raised by the emphasis on body image in Chinese apps, we suggest that designs should still leverage persuasive principles to convey scientific best practices, nudging people's attitudes and behaviors to be healthier. Therefore, our answer to the question above is: for health \& wellness technologies, prioritizing health over cultural norms is important, especially when the cultural norm is not supported by scientific knowledge. More specifically, we argue that we can leverage persuasive design to reinforce people's cultural perception when it aligns with scientific knowledge, but we have a responsibility as designers aim to change or shape people's perception when culture conflicts with science.

This tension also brings out ethical issues for us to consider as researchers and designers. Culturally appropriate persuasive designs could more strongly convince people and sustain their interaction within the app, improving app effectiveness, stickiness, and ultimately profit. Throughout the interview, our participants also mentioned: "[these persuasive features] can increase users' attachment to the app and thus generate cash flow, which is quite common in internet companies" (P10m). However, our study suggests that cultural norms could also be potentially risky and lead to serious consequences for people's health \& wellness. Therefore, when designing culturally appropriate persuasive systems, we should consider the balance between commercial profits and individual benefits by understanding the impact of our design decisions on individual's health behaviors.

We also point out a fundamental tension about the inclusion of cultural norms in technology that designs inherently cannot satisfy everyone given the diversity of cultural norms. As researchers and 
designers, we should seek to understand people's opinions about a cultural norm, and try to achieve a balance among following divergent cultural norms in design.

\subsection{Design Opportunities}

The goal of this paper is not to conclude whether it is "better" or "worse" that Chinese food journaling apps leverage persuasive design strategies to emphasize body image while Western apps implement the same strategies to prioritize people's health through managing weight. Instead, we aim to draw attention how food journaling apps can reinforce culturally-constructed principles around body image. Body image is a complex topic closely intertwined with various media technologies, such as the social media and TV shows [67]. People's experiences related to body image have also been shaped by their lived experiences. Therefore, it is difficult to subvert the Chinese cultural norm of beauty and change people's perceptions toward body image by just improving the current design of Chinese food journaling apps. However, the findings of this study indicate some potential design opportunities about how to critically leverage persuasive design principles in Chinese food journaling apps regarding body image. Food journaling app should aim to leverage persuasive design more inclusively, while still understanding cultural norms to be able to appropriately support people's goals while nudging them to a healthier direction.

6.3.1 Utilizing persuasive design to promote healthy and inclusive perceptions toward body image. Rather than completely removing design elements related to body image, we suggest that technology could emphasize body image differently to make Chinese apps more inclusive and healthier. Inspired by Western apps' prioritizing health and only utilizing body image in workout tutorials, the implementation of persuasive design strategies in Chinese apps could focus on appreciating a healthy body shape instead of a "perfect" one. For example, Chinese apps tend to leverage success stories and attractive models to highlight the importance of obtaining a perfect and slim body shape. To promote healthy body image in Chinese apps, the success stories could instead accentuate the health benefits people achieve once they lose weight, and more diverse body shapes could be incorporated.

In addition, the persuasive design category of system credibility was rarely used to emphasize body image in Chinese apps. However, some principles under this category, such as expertise and authority, could be used to help gradually construct a healthier perception towards body image. These principles can be applied to teach people scientific knowledge about what a healthy body shape is and how to obtain it. These principles can also educate people about other beauty norms, making them have more open-minded attitudes toward different conceptions of beauty. For example, Chinese food journaling apps could invite nutritionists, psychological experts, or professional coaches to write articles, such as what healthy body shape can be, how to build body positivity, and the risks of simply pursuing thinness. Apps could also often introduce articles about health \& wellness from the websites of Chinese public health departments, such as the Chinese Nutrition Society, to describe alternate conceptions of healthy weights and bodies.

We see benefits in both Chinese and Western food journaling apps focusing less on weight and calorie-counting, following the personalization persuasive principle to adjust the app's focus on weight to people's motivations for journaling. For people who aim to build a healthier eating habit or people who monitor their food for chronic health conditions (except weight related), the app can de-emphasize these data in favor of other monitoring information.

6.3.2 Rethinking food and body image. Controlling diet has been a major approach for people in China to lose weight [2] and some participants in our study aimed to leverage the food journaling app to reach a goal of being underweight. Therefore, building a healthier eating behavior is also crucial for constructing a healthier perception towards body image. As Grimes and Harper [34] suggested to regard food as a celebratory space, persuasive design principles can be also used to help people recognize a positive and even celebratory relationship between healthy food and healthy body shape. For example, the number-driven goal setting of food journaling apps is critiqued for making people become too enthusiastic about counting calories [32]. Accordingly, persuasive design features such as goal-setting and feedback, could move beyond just calories and help people identify other things that matter to eating, such as how they feel and what benefits that consuming certain foods would bring to their health and body shape.

Current food journaling apps often leverage the feedback persuasive principle by providing macronutrient analysis, such as how much protein, fat, and carbs that a person has eaten. However, this persuasive feedback usually is too "datafied" and normal users are not data scientists. Food journaling apps could provide more direct and easy-to-understand feedback about other nutrition benefits of food to aim to persuade participants towards a more holistic perspective on health, and to connect nutrients to aspects of beauty a person might aim to prioritize. For example, the food journaling app could say: "Wow, the blueberry you just eat will reward you with a stronger heart! It also contains antioxidants that can make your skin better".

Reframing food and body image likely requires intervention beyond individual apps or designs. Learning from how other work on promoting help has approached "leveling up" is important towards reframing cultural conversations around food [71]. For example, exploring how technology can help reframe food and body eating conversations in education can result in larger adjustments to cultural perception.

\subsection{Limitations}

At the time of our study, food journaling apps in China were not as prevalent as they were in Western countries. Chinese food journaling apps have much fewer downloads than Western apps. For example, MyFitnessPal has received over 1 million ratings in the U.S App Store while the most popular food journaling app in China, Mint, has only 100,000 ratings in the Chinese App Store. Therefore, our findings mainly reflect the early and emergent themes in the current Chinese food journaling apps. If food journaling becomes as popular in China as it is in the Western culture, the focus on beauty and these corresponding persuasive strategies may change to account for people with diverse journaling motivations.

We examined and compared the most popular food journaling apps in China and the West to understand common persuasive features that people tend to encounter when using a food journaling 
app. However, other apps that we did not examine might leverage persuasive strategies differently, such as Western apps which heavily emphasize cultural norms around beauty. Therefore, rather than regarding our findings as a generalization, they are more like an indication of the most widely-used persuasive techniques adopted by Chinese and Western apps.

Our interview participants are relatively young, nearly all being in their 20's. Their opinions may not reflect those of people in other age ranges. People older than our participants may have different perceptions of cultural beauty norms and their influence on people's motivations for food journaling, and particularly on how culture should manifest in technology. Our interviews did not obtain participant feedback on all persuasive features that emphasized body image, as our interview findings led us to conduct a thorough feature analysis of how body image is represented in Chinese and Western apps. We introduce points where our participants mentioned their experience using some of these features, but further work could provide additional insight into participant perspectives. Relatedly, participant perspectives should not be regarded as a summative characterization of their thoughts on the specific apps we studied. Although participants offered their thoughts on other features of the apps they had used, our interview mainly focused on persuasive related to body image.

Though our study mainly attributes Chinese food journaling apps' emphasis on body image to the single-dimensional cultural beauty norm, other cultural differences likely influence the design. For example, the obesity rate in China is about 6\% (though it has increased in recent years [14]) compared to over $36 \%$ in the United States where many of the Western apps are popular [64]. Therefore, food journaling apps in Western countries may prioritize health over beauty due to facing more serious public health concerns.

\section{CONCLUSION}

In examining the influence of cultural norms on the design of Chinese food journaling apps, we find that Chinese apps leverage persuasive designs strategies to portray an ideal body shape and encourage the pursuit towards thinness while Western apps utilize similar strategies to emphasize a healthy diet or weight. Aligned with Chinese cultural norms around beauty, most of our participants embraced Chinese apps' emphasis on body image, though they indicated that this emphasis could lead those who did not align with this cultural perception to feel excluded. Rather than subverting the cultural norm of beauty in designing Chinese food journaling apps, we suggest that designs can critically apply persuasive design principles to help people pursue healthier and more inclusive goals. In addition, persuasive design principles can be used to build a positive and celebratory relationship between healthy eating and a healthy body shape.

\section{ACKNOWLEDGMENTS}

We thank our anonymous reviewers for their feedback. This work was funded in part by the National Science Foundation under award IIS-1850389.

\section{REFERENCES}

[1] •E-Health Application Categories Used by U.S. Adults 2017 | Statista. https://www.statista.com/statistics/378850/top-mobile-health-application- categories-used-by-us-consumers/

[2] 2013 Chinese Internet Users Body Weight Report. http://fitness.39.net/report2013/2013中国网民健康体重调查报告.pdf

[3] Teresa Almeida, Rob Comber, Gavin Wood, Dean Saraf, and Madeline Balaam. (2016). On Looking at the Vagina through Labella. Proceedings of the SIGCHI Conference on Human Factors in Computing Systems (CHI 2016), ACM. http://doi. org $/ 10.1145 / 2858036.2858119$

[4] Average Human Height by Country - Wikipedia. https://en.wikipedia.org/wiki/ Average_human_height_by_country

[5] Arda Aydin, Anthony Micallef, Samantha Lovelace, Xudong Li, Victor Cheung, and Audrey Girouard. (2017). Save the Kiwi: Encouraging Better Food Management through Behaviour Change and Persuasive Design Theories in a Mobile App Late-Breaking Work. Proceedings of the SIGCHI Conference Extended Abstracts on Human Factors in Computing Systems (CHI EA 2017), ACM. http://doi.org/10.1145/3027063.3053192

[6] Amid Ayobi, Paul Marshall, Anna L. Cox, and Yunan Chen. (2017). Quantifying the Body and Caring for the Mind: Self-Tracking in Multiple Sclerosis. Proceedings of the SIGCHI Conference on Human Factors in Computing Systems (CHI 2017), ACM. http://doi.org/10.1145/3025453.3025869

[7] Andrea M. Barbarin, Laura R. Saslow, Mark S. Ackerman, and Tiffany C. Veinot. (2018). Toward Health Information Technology that Supports Overweight/Obese Women in Addressing Emotion- and Stress-related Eating. Proceedings of the SIGCHI Conference on Human Factors in Computing Systems (CHI 2018). https: //doi.org/10.1145/3173574.3173895

[8] Body Fat Percentage - Wikipedia. https://en.wikipedia.org/wiki/Body_fat_ percentage

[9] Virginia Braun and Victoria Clarke. (2012). Thematic Analysis. In APA handbook of research methods in psychology, Vol 2: Research designs: Quantitative, qualitative, neuropsychological, and biological. American Psychological Association, 57-71. http://doi.org/10.1037/13620-004

[10] Lora E. Burke, Mindi A. Styn, Susan M. Sereika, Molly B. Conroy, Lei Ye, Karen Glanz, Mary Ann Sevick, and Linda J. Ewing. (2012). Using mHealth Technology to Enhance Self-Monitoring for Weight Loss: A Randomized Trial. American fournal of Preventive Medicine, 43(1), 20-26. http://doi.org/10.1016/j.amepre.2012.03.016

[11] CDC. Session 7: Tip the Calorie Balance National Diabetes Prevention Program 1 Quick Fact Session 7: Overview About Calories Energy Use Through Activity. https://www.cdc.gov/diabetes/prevention/pdf/handout_session7.pdf

[12] Stevie Chancellor, Jessica Annette Pater, Trustin Clear, Eric Gilbert, and Munmun De Choudhury. (2016). \#Thyghgapp: Instagram Content Moderation and Lexical Variation in Pro-Eating Disorder Communities. Proceedings of the ACM Conference on Computer Supported Cooperative Work, CSCW (CSCW 2016), 1201-1213. http: //doi.org/10.1145/2818048.2819963

[13] Change on the App Store. https://apps.apple.com/us/app/变啦-糖脂代谢管理平 台/id1173327490

[14] C. M. Chen. (2008). Overview of Obesity in Mainland China. Obesity Reviews, 9(s1), 14-21. http://doi.org/10.1111/j.1467-789X.2007.00433.x

[15] Boreum Choi, Inseong Lee, Jinwoo Kim, and Yunsuk Jeon. (2005). A Qualitative Cross-National Study of Cultural Influences on Mobile Data Service Design. Proceedings of the SIGCHI Conference on Human Factors in Computing Systems (CHI 2005), ACM Press. http://doi.org/10.1145/1054972.1055064

[16] Chia-Fang Chung, Elena Agapie, Jessica Schroeder, Sonali Mishra, James Fogarty, and Sean A. Munson. (2017). When Personal Tacking Becomes Social: Examining the Use of Instagram for Healthy Eating. Proceedings of the SIGCHI Conference on Human Factors in Computing Systems (CHI 2017), 1674-1687. https://doi.org/10. $1145 / 3025453.3025747$

[17] Felicia Cordeiro, Elizabeth Bales, Erin Cherry, and James Fogarty. (2015). Rethinking the Mobile Food Journal: Exploring Opportunities for Lightweight Photo-Based Capture. Proceedings of the SIGCHI Conference on Human Factors in Computing Systems (CHI 2015), ACM. http://doi.org/10.1145/2702123.2702154

[18] Felicia Cordeiro, Daniel A. Epstein, Edison Thomaz, Elizabeth Bales, Arvind K. Jagannathan, Gregory D. Abowd, and James Fogarty. (2015). Barriers and Negative Nudges: Exploring Challenges in Food Journaling. Proceedings of the SIGCHI Conference on Human Factors in Computing Systems (CHI 2015), ACM. http://doi.org/10.1145/2702123.2702155

[19] Criteria of Weight for Adults. http://www.nhc.gov.cn/ewebeditor/uploadfile/ 2013/08/20130808135715967.pdf

[20] Cindy Davis and Melanie A. Katzman. (1998). Chinese Men and Women in the United States and Hong Kong: Body and Self-Esteem Ratings as a Prelude to Dieting and Exercise. International fournal of Eating Disorders, 23(1), 99-102. http: //doi.org/10.1002/(SICI)1098-108X(199801)23:1<99::AID-EAT13>3.0.CO;2-I

[21] Elizabeth V. Eikey, Yunan Chen, and Kai Zheng. (2019). Do Recovery Apps Even Exist?: Why College Women with Eating Disorders Use (But Not Recommend) Diet and Fitness Apps Over Recovery Apps. Lecture Notes in Computer Science (including subseries Lecture Notes in Artificial Intelligence and Lecture Notes in Bioinformatics), Springer Verlag, 727-740. http://doi.org/10.1007/978-3-030-15742569

[22] Elizabeth V. Eikey and Madhu C. Reddy. (2017). "It's Definitely Been a Journey": A Qualitative Study on How Women with Eating Disorders Use Weight Loss Apps. 
Proceedings of the SIGCHI Conference on Human Factors in Computing Systems (CHI 2017), ACM. http://doi.org/10.1145/3025453.3025591.

[23] Elizabeth V. Eikey, Madhu C. Reddy, Kayla M. Booth, Lynette Kvasny, Johnna L. Blair, Victor Li, and Erika S. Poole. (2017). Desire to be Underweight: Exploratory Study on a Weight Loss App Community and User Perceptions of the Impact on Disordered Eating Behaviors. FMIR mHealth and uHealth, 5(10), e6683. http //doi.org/10.2196/mhealth.6683

[24] Daniel A. Epstein, Clara Caldeira, Mayara Costa Figueiredo, Xi Lu, Lucas M. Silva, Lucretia Williams, Jong Ho Lee, Qingyang Li, Simran Ahuja, Qiuer Chen, Payam Dowlatyari, Craig Hilby, Sazeda Sultana, Elizabeth V. Eikey, and Yunan Chen (2020). Mapping and Taking Stock of the Personal Informatics Literature. Proceedings of the ACM on Interactive, Mobile, Wearable and Ubiquitous Technologies, 4(4), 1-38. http://doi.org/10.1145/3432231

[25] Daniel A. Epstein, Felicia Cordeiro, James Fogarty, Gary Hsieh, and Sean A. Munson. (2016). Crumbs: Lightweight Daily Food Challenges to Promote Engagement and Mindfulness. Proceedings of the SIGCHI Conference on Human Factors in Computing Systems (CHI 2016), 5632-5644. https://doi.org/10.1145/2858036.2858044

[26] Anthony Faiola and Sorin A. Matei. (2005). Cultural Cognitive Style and Web Design: Beyond a Behavioral Inquiry into Computer-Mediated Communication. Journal of Computer-Mediated Communication, 11(1), 375-394. http://doi.org/10. 1111/j.1083-6101.2006.tb00318.x

[27] Giannina Ferrara, Jenna Kim, Shuhao Lin, Jenna Hua, and Edmund Seto. (2019). A Focused Review of Smartphone Diet-Tracking Apps: Usability, Functionality, Coherence with Behavior Change Theory, and Comparative Validity of Nutrient Intake and Energy Estimates. FMIR mHealth and uHealth, 7(5), e9232. http://doi. org $/ 10.2196 /$ mhealth. 9232

[28] Marta Ferrer-García and José Gutiérrez-Maldonado. (2012). The Use of Virtual Reality in the Study, Assessment, and Treatment of Body Image in Eating Disorders and Nonclinical Samples: A Review of the Literature. Body Image 9, 1-11. http://doi.org/10.1016/j.bodyim.2011.10.001

[29] Katherine M. Flegal, Barry I. Graubard, David F. Williamson, and Mitchell H. Gail. (2005). Excess Deaths Associated with Underweight, Overweight, and Obesity. Fournal of the American Medical Association, 293(15), 1861-1867. http://doi.org/ 10.1001/jama.293.15.1861

[30] B. J. Fogg. (2002). Persuasive Technology: Using Computers to Change What We Think and Do. Ubiquity, 2002(December), 2. http://doi.org/10.1145/764008.763957

[31] B. J. Fogg. (2019). Tiny Habits: The Small Changes That Change Everything. Eamon Dolan Books.

[32] Thomas Fritz, Elaine M. Huang, Gail C. Murphy, and Thomas Zimmermann (2014). Persuasive Technology in the Real World: A Study of Long-Term Use of Activity Sensing Devices for Fitness. Proceedings of the SIGCHI Conference on Human Factors in Computing Systems (CHI 2014), ACM. http://doi.org/10.1145/ 2556288.2557383

[33] Kathrin M. Gerling, Regan L. Mandryk, Max Birk, Matthew Miller, and Rita Orji. (2014). The Effects of Embodied Persuasive Games on Player Attitudes Toward People Using Wheelchairs. Proceedings of the SIGCHI Conference on Human Factors in Computing Systems (CHI 2014), ACM. http://doi.org/10.1145/2556288.2556962

[34] Andrea Grimes and Richard Harper. (2008). Celebratory Technology: New Directions for Food Research in HCI. Proceedings of the SIGCHI Conference on Human Factors in Computing Systems (CHI 2008), 467-476. https://doi.org/10. 1145/1357054.1357130

[35] Shathel Haddad, Joanna Mcgrenere, and Claudia Jacova. (2014). Interface Design for Older Adults with Varying Cultural Attitudes toward Uncertainty. Proceedings of the SIGCHI Conference on Human Factors in Computing Systems (CHI 2014), ACM. http://doi.org/10.1145/2556288.2557124

[36] Brit Harper and Marika Tiggemann. (2008). The Effect of Thin Ideal Media Images on Women's Self-Objectification, Mood, and Body Image. Sex Roles, 58(9-10), 649-657. http://doi.org/10.1007/s11199-007-9379-x

[37] Mahsa Honary, Beth T. Bell, Sarah Clinch, Sarah E. Wild, and Roisin McNaney. (2019). Understanding the Role of Healthy Eating and Fitness Mobile Apps in the Formation of Maladaptive Eating and Exercise Behaviors in Young People. FMIR mHealth and uHealth, 7(6), e14239. http://doi.org/10.2196/14239

[38] Anne Hsu, Jing Yang, Yigit Yilmaz, Sanaul Haque, Cengiz Can, and Ann Blandford. (2014). Persuasive Technology for Overcoming Food Cravings and Improving Snack Choices. Proceedings of the SIGCHI Conference on Human Factors in Computing Systems (CHI 2014), ACM. http://doi.org/10.1145/2556288.2557099

[39] Jr Jussaume. (2001). Factors Associated with Modern Urban Chinese Food Consumption Patterns. Fournal of Contemporary China, 10(27), 219-232. http: //doi.org/10.1080/10670560120045715

[40] Ravi Karkar, Jessica Schroeder, Daniel A. Epstein, Laura R. Pina, Jeffrey Scofield, James Fogarty, Julie A. Kientz, Sean A. Munson, Roger Vilardaga, and Jasmine Zia. (2017). TummyTrials: A Feasibility Study of Using Self-Experimentation to Detect Individualized Food Triggers. Proceedings of the SIGCHI Conference on Human Factors in Computing Systems (CHI 2017), Association for Computing Machinery, 6850-6863. http://doi.org/10.1145/3025453.3025480

[41] M. Alexis Kennedy, Laura Templeton, Anita Gandhi, and Boris B. Gorzalka. (2004) Asian Body Image Satisfaction: Ethnic and Gender Differences across Chinese, Indo-Asian, and European-Descent Students. Eating Disorders, 12(4), 321-336. http://doi.org/10.1080/10640260490521415

[42] Kyung Bo Kim. (2014). Narratives about the Media, Diet, and Body Image: A Cross-Cultural Comparison between Young Female Adults in the Midwestern United States and South Korea. Fournal of Intercultural Communication Research, 43(4), 283-303. http://doi.org/10.1080/17475759.2014.952768

[43] Sing Lee, Tony Leung, Antoinette M. Lee, Hong Yu, and C. M. Leung. (1996). Body Dissatisfaction among Chinese Undergraduates and Its Implications for Eating Disorders in Hong Kong. International fournal of Eating Disorders, 20(1), 77-84. http://doi.org/10.1002/(SICI)1098-108X(199607)20:1<77::AID-EAT9>3.0.CO;2-1

[44] Cheri A. Levinson, Laura Fewell, and Leigh C. Brosof. (2017). My Fitness Pal Calorie Tracker Usage in the Eating Disorders. Eating Behaviors, 27, 14-16. http: //doi.org/10.1016/j.eatbeh.2017.08.003

[45] Jian-rong Li and Yun-Hwa P Hsieh. (2004). Traditional Chinese Food Technology and Cuisine. Asia Pac f Clin Nutr, 13(2), 147-155. https://pubmed.ncbi.nlm.nih. gov/15228981/

[46] Lose It! - Calorie Counter on the App Store. https://apps.apple.com/us/app/loseit-calorie-counter/id297368629

[47] Losing Weight $\mid$ Healthy Weight, Nutrition, and Physical Activity | CDC. https: //www.cdc.gov/healthyweight/losing weight/index.html

[48] Valeria Lotti. (2018). The Image of the Beautiful Woman: Beauty Ideals in Modern Urban China. ASIEN, 147(April), 92-104.

[49] Kai Lukoff, Taoxi Li, Yuan Zhuang, and Brian Y. Lim. (2018). TableChat: Mobile Food Journaling to Facilitate Family Support for Healthy Eating. Proceedings of the ACM on Human-Computer Interaction, 2(CSCW), 114. https://doi.org/10.1145/ 3274383

[50] Yuhan Luo, Peiyi Liu, and Eun Kyoung Choe. (2019). Co-designing Food Trackers with Dietitians: Identifying Design Opportunities for Food Tracker Customization. Proceedings of the SIGCHI Conference on Human Factors in Computing Systems (CHI 2019). https://doi.org/10.1145/3290605.3300822

[51] Deborah Lupton. (2017). Digital Media and Body Weight, Shape, and Size: An Introduction and Review. Fat Studies, 6(2), 119-134. http://doi.org/10.1080/21604851. 2017.1243392

[52] Guansheng Ma. (2015). Food, Eating Behavior, and Culture in Chinese Society. Journal of Ethnic Foods, 2(4), 195-199. http://doi.org/10.1016/j.jef.2015.11.004

[53] Aaron Marcus. (2001). Cross-Cultural User-Interface Design for Work, Home, Play, and On the Way. Proceedings of the international conference on Computer documentation (SIGDOC 2001), ACM Press. http://doi.org/10.1145/1900520.1900525

[54] David Mellor, Monique Waterhouse, Norul Hidayah bt Mamat, Xiaoyan Xu, Jamie Cochrane, Marita McCabe, and Lina Ricciardelli. (2013). Which Body Features are Associated with Female Adolescents' Body Dissatisfaction? A Cross-Cultural Study in Australia, China and Malaysia. Body Image, 10(1), 54-61. http://doi.org/ 10.1016/j.bodyim.2012.10.002

[55] Mint on the App Store. https://apps.apple.com/cn/app/薄荷健康-减肥健身运动 减脂/id457856023

[56] More Needs to Promote Nutrition Knowledge When the Healthy Weight Loss Becomes Mainstream. http://www.chinacdc.cn/mtbd_8067/201511/t20151109_ 121794.html

[57] MyFitnessPal.com. (2017). MyFitnessPal on the App Store. https://apps.apple. com/us/app/myfitnesspal/id341232718

[58] Harri Oinas-Kukkonen and Marja Harjumaa. (2009). Persuasive Systems Design: Key Issues, Process Model, and System Features. Communications of the Association for Information Systems, 24(1), 485-500. http://doi.org/10.17705/1cais.02428

[59] Rita Orji, Lennart E. Nacke, and Chrysanne Di Marco. (2017). Towards PersonalityDriven Persuasive Health Games and Gamified Systems. Proceedings of the SIGCHI Conference on Human Factors in Computing Systems (CHI 2017), ACM. http://doi. org/10.1145/3025453.3025577

[60] Kiemute Oyibo, Ifeoma Adaji, Rita Orji, Babatunde Olabenjo, Mahsa Azizi, Julita Vassileva, K Oyibo, I Adaji, R Orji, B Olabenjo, M Azizi, and J Vassileva. (2018). Perceived Persuasive Effect of Behavior Model Design in Fitness Apps. Proceedings of the 26th Conference on User Modeling, Adaptation and Personalization, ACM, 10. https://doi.org/10.1145/3209219.3209240

[61] Jessica A. Pater, Oliver L. Haimson, Nazanin Andalibi, and Elizabeth D. Mynatt. (2016). "Hunger Hurts but Starving Works:" Characterizing the Presentation of Eating Disorders Online. Proceedings of the ACM Conference on Computer Supported Cooperative Work, CSCW (CSCW 2016), ACM. http://doi.org/10.1145/ 2818048.2820030

[62] Tom Plocher, Rau Pei-Luen Patrick, and Choong Yee-Yin. (2012). Cross-Cultural Design. In Handbook of Human Factors and Ergonomics. 162-191. http://doi.org/ 10.1002/9781118131350.ch6

[63] Stephen Purpura, Victoria Schwanda, Kaiton Williams, William Stubler, and Phoebe Sengers. (2011). Fit4Life: The Design of a Persuasive Technology Promoting Healthy Behavior and Ideal Weight. Proceedings of the SIGCHI Conference on Human Factors in Computing Systems (CHI 2011), ACM Press. http: //doi.org/10.1145/1978942.1979003

[64] Ranking (\% obesity by country), 2016 | World Obesity Federation Global Obesity Observatory. https://data.worldobesity.org/rankings/

[65] Katharina Reinecke and Abraham Bernstein. (2011). Improving Performance, Perceived Usability, and Aesthetics with Culturally Adaptive User Interfaces. 
ACM Transactions on Computer-Human Interaction, 18(2), 1-29. http://doi.org/10. 1145/1970378.1970382

[66] Ruka Sakamaki, Kenji Toyama, Rie Amamoto, Chuan-Jun Liu, and Naotaka Shinfuku. (2005). Nutritional Knowledge, Food Habits and Health Attitude of Chinese University Students-a Cross Sectional Study. http://doi.org/10.1186/14752891-4-4

[67] David Šmahel, Hana Macháčková, Martina Šmahelová, Michal Čevelíček, Carlos A. Almenara, Jana Holubčíková, David Šmahel, Hana Macháčková, Martina Šmahelová, Michal Čevelíček, Carlos A. Almenara, and Jana Holubčíková. (2018). Technology, Body Image, and Disordered Eating. In Digital Technology, Eating Behaviors, and Eating Disorders. Springer International Publishing, 65-82. http: //doi.org/10.1007/978-3-319-93221-7_4

[68] Piya Sorcar, Benjamin Strauber, Prashant Loyalka, Neha Kumar, and Shelley Goldman. (2017). Sidestepping the Elephant in the Classroom: Using Culturally Localized Technology to Teach Around Taboos. Proceedings of the SIGCHI Conference on Human Factors in Computing Systems (CHI 2017), ACM. http: //doi.org/10.1145/3025453.3025958

[69] The Average Weight of Chinese Adult Male and Female are $69.6 \mathrm{~kg}$ and $59 \mathrm{~kg}$ Respectively. https://www.chinanews.com/gn/2020/12-23/9369096.shtml

[70] Callie Thomson, Jane Nash, and Anthony Maeder. (2016). Persuasive Design for Behaviour Change Apps: Issues for Designers. Proceedings of the Annual Conference of the South African Institute of Computer Scientists and Information Technologists on (SAICSIT 2016), ACM Press. http://doi.org/10.1145/2987491.2987535
[71] Tiffany C. Veinot, Jessica S. Ancker, Heather Cole-Lewis, Elizabeth D. Mynatt, Andrea G. Parker, Katie A. Siek, and Lena Mamykina. (2019). Leveling Up. Medical Care, 57(Suppl 2), S108-S114. http://doi.org/10.1097/MLR.0000000000001032

[72] Vanissa Wanick, Ashok Ranchhod, and Gary Wills. (2015). Cultural Persuasive Affordances in Advergaming Design Across Cultures: a Conceptual Model. Proceedings of the International Academic Mindtrek Conference (MindTrek 2015), ACM. http://doi.org/10.1145/2818187.2818293

[73] Weight Loss Secretary on the App Store. https://apps.apple.com/us/app/减肥小 秘书-减肥打卡食物热量计算/id520726724

[74] Yan Yan and Kim Bissell. (2014). The Globalization of Beauty: How is Ideal Beauty Influenced by Globally Published Fashion and Beauty Magazines? Journal of Intercultural Communication Research, 43(3), 194-214. http://doi.org/10.1080/ 17475759.2014.917432

[75] Lei Zhang, Haihong Oian, and Hua Fu. (2018). To Be Thin But Not Healthy - The Body-Image Dilemma May Affect Health Among Female University Students in China. PLOS ONE, 13(10), e0205282. http://doi.org/10.1371/journal.pone.0205282

[76] Meng Zhang. (2012). A Chinese Beauty Story: How College Women in China Negotiate Beauty, Body Image, and Mass Media. Chinese fournal of Communication, 5(4), 437-454. http://doi.org/10.1080/17544750.2012.723387 